\title{
Sobre las geometrías no euclidianas: Notas históricas y bibliográficas
}

Francisco José Duarte Isava

Rev. Acad. Colomb. Cienc. Ex. Fis. Nat. 1946, 7 (25-26): 63-81.

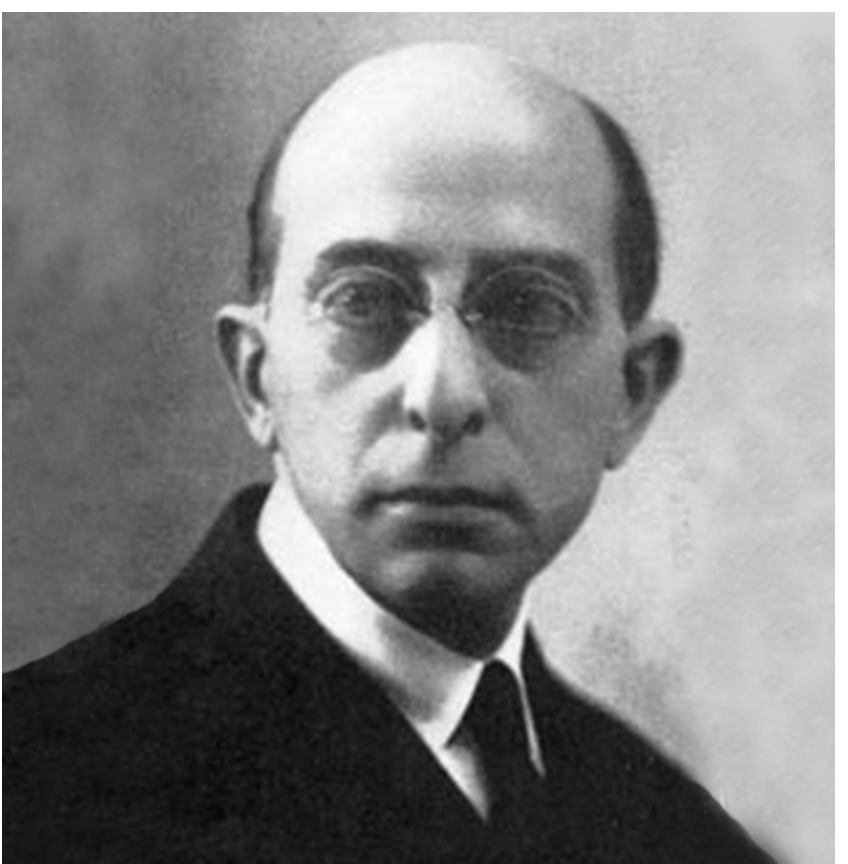

\section{Francisco José Duarte Isava (1883-1972)}

Ingeniero venezolano reconocido como uno de los científicos más importantes de su país. Alternó su trabajo como ingeniero con la docencia en la Universidad Central de Venezuela, como profesor de geometría analítica, algebra superior, análisis infinitesimal y mecánica racional, y también de Algebra Superior en la Universidad Santa María, en Caracas. En 1933 funda la Academia de Ciencias Físicas, Matemáticas y Naturales de Venezuela, institución que presidió en dos ocasiones. Fue además director del Observatorio Astronómico y Meteorológico “Juan Manuel Cagigal”. Participó activamente en los Encuentros Internacionales de Matemáticos de Bologna (1928), Zurich (1932) y Boston (1950) y en varios Congresos de la Unión Geodésica y Geofísica Internacional, así como en los congresos internacionales para el uso pacífico de la energía atómica, en Ginebra en 1955 y 1958. Publicó 7 libros, más de 80 trabajos matemáticos en revistas de renombre internacional, y escribió, adicionalmente, una gran cantidad de notas de prensa que incluyen biografías de matemáticos famosos. Recibió muchos reconocimientos en Venezuela por su distinguida labor en pro de la ciencia y la matemática en su país.

Información biográfica suplementaria: http://www.tayabeixo. org/biografias/duarte.htm
Duarte Isava escribió un extenso y bien documentado artículo sobre la historia del postulado de Euclides y el surgimiento de las geometrías no euclidianas. Dedicó un apartado a los Ensayos de demostración del postulado de Euclides del Dr. Julio Garavito Armero (1865-1920), en el cual hace un riguroso análisis de dos demostraciones de Garavito para concluir - en el caso de la primera- que este ejericio es un claro ejemplo de los ensayos de demostración en los cuales se reemplaza el postulado de Euclides por otro más difícil de admitir. Sobre el segundo ensayo afirma que el error de Garavito está en haber olvidado que los teoremas de la Geometría no son verdades geométricas sino verdades enunciadas en un lenguaje geométrico, las cuales no se aplican a figuras concretas de la geometría mientras no se acuerden previamente los postulados fundamentales. En otros términos, que las verdades de la geometría no son absolutas sino relativas al sistema de principios en el cual se basa. Este trabajo le sirvió a Duarte para ingresar como miembro correspondiente a la Academia Colombiana de Ciencias Exactas, Físicas y Naturales ${ }^{1}$. Dos aspectos hacen muy valioso este artículo para la historia de las matemáticas en Colombia: la rigurosidad del mismo y las fuentes utilizadas, además de la Nota Editorial ${ }^{2}$ que aparece al final del artículo, en la cual Álvarez Lleras manifiesta que en otra ocasión probará que Duarte está equivocado, "porque Garavito, más que matemático, fue filósofo sincero que nunca tuvo en mente cosa diferente de la persecución de la verdad" y "así haremos ver que los errores que el doctor Duarte cree encontrar en las exposiciones de Garavito, no lo son para todos, por cuanto la escuela de los pangeómetras no es universal, ni las ideas contrarias a la matemática clásica han obtenido hasta ahora un triunfo absoluto.” La corrección a Duarte nunca apareció.

Clara Helena Sánchez, Ph.D.

Miembro Correspondiente

${ }^{1}$ Aunque había sido publicado en 1945 en el Boletín de la Academia de Ciencias Físicas Matemáticas y Naturales de Venezuela, Año XI, Tomo IX, No.26, pp.3-52.

${ }^{2}$ Nota de la Dirección, Rev, Acad. Colomb, Cienc. , Vol. VII, Nos. 25-26, p.81. 


\title{
SOBRE LAS GEOMETRIAS NO EUCLIDIANAS
}

\section{NOTAS HISTORICAS Y BIBLIOGRAFICAS}

\author{
F. J. DUARTE \\ Miembro Correspondiente de la Academia Colombiana \\ de Ciencias Exactas, Físico-Químicas y Naturales.
}

\section{INTRODUCCION}

El descubrimiento o, si se quiere, la creación de la Geometría no-euclidiana antes de terminar el primer tercio del siglo XIX es un acontecimiento trascendental en la historia de las Matemáticas. Como ha sucedido muchas veces en casos análogos, este gran descubrimiento no fue entendido por los contemporáneos de Lobatchevsky: "Tal como era parecía contradecir un axioma cuya necesidad está basada únicamente sobre un prejuicio consagrado desde hace millares de años", como dicen los editores de las obras del ilustre geómetra. "Se necesitaron todavía cien años - dice Gonseth - para que, con Poincaré y Einstein, nos diéramos cuenta de la trascendencia de este descubrimiento". [124], 79.

Cuenta el historiador y matemático Eric Bell que Lobatchowsky pasó cuarenta años en la Universidad de Kasan, como estudiante, profesor adjunto, profesor y finalmente rector. Los servicios eminentes que prestó a su patria tuvieron por única recompensa que el gobierno ruso lo destituyera bruscamente en 1846 de sus funciones de rector $y$ profesor de la Universidad, "sin dar ninguna explicación pública de este doble insulto inmerecido" [161], $325\left(^{*}\right)$. Los otros profesores de la Universidad, arriesgando su propia situación, protestaron unánimemente contra este ultraje. Se les respondió secamente que, "como simples profesores no tenian capacidad para juzgar los actos del Gobierno". No era la primera vez que ocurría en el mundo semejante injusticia: recuérdese, entre otras, la historia de Tycho Brahe.

Nueve años después, en 1855 , la Universidad de Kasan celebraba el primer medio siglo de existencia. Lobatchewsky concurrió a la conmemoración y presentó un ejemplar de su inmortal Pangeometría, resumen del trabajo completo de su vida científica. Pocos meses más tarde Lobatchewsky murió a la edad de 63 años.

Como muy bien ha dicho Parfentieff: "A medida que el pensamiento matemático y filosófico moderno profundiza sus problemas actuales, nos persuadimos más de que diversos pensamientos, ideas y métodos de Lobatchewsky, ese genio potente y profundo, penetran en todas las ramas de las ciencias físico-matemáticas, tienen influencia sobre su desarrollo y vemos mejor qué gran valor tiene y seguirá teniendo la Geometría no-euclidiana de Lobatchewsky en general en la filosofía de la Naturaleza". [150], 476.

(*) Los números entre paréntesis indican la obra correspondiente en la bibliografía al fin de este trabajo; el número colocado al lado indíca la página del libro citado.
El 26 de febrero de 1926 la Sociedad Físico-Matemática y la Universidad de Kasan celebraron, en presencia de numerosos delegados oficiales, el centenario del descubrimiento de la geometría no euclidiana por Lobatchewsky. La Sociedad Físico-Matemática de Kasan publicó en esta ocasión un volumen cuyo título es:

"Ad anum MCMXXVI centesimum a geometra Kasaniensi N. J. Lobacevsky non-euklidae geometriae systematis inventi concelebrandum". In $8^{\circ}$ de 112 pág. 1927.

Nuestro querido y venerado maestro, el ilustre matemático Dimitry Mirimanoff, al hacer el análisis de esta publicación, termina con estas palabras: (")

"Víctor Hugo dijo con ocasión del $6^{\circ}$ centenario del Dante: 'Una solemnidad como esta es un magnífico síntoma. Es la fiesta de todos los hombres celebrada por una nación como homenaje a un genio. Cada nación da a las otras una parte de su grande hombre. La unión de los pueblos se prepara por la fraternidad de los genios'.

";No se podrían aplicar estas palabras al centenario de uno de los más grandes descubrimientos del siglo XIX?".

El objeto del trabajo que hoy sale a luz es, como lo indica su título, presentar algunas notas históricas y bibliográficas sobre las geometrías no-euclidianas. Ni las unas ni las otras pretendemos que puedan ser completas.

También exponemos varias de las numerosas demostraciones que se ha pretendido dar del postulado de Euclides, con la crítica correspondiente.

Existen varias listas bibliográficas de las geometrías no-euclidianas, las que citamos en la Bibliografía al final de este trabajo [13], [34,] [39], [69]. No conocemos ninguna de estas listas; nuestra bibliografía está formada por las obras que poseemos en nuestra biblioteca particular y por los libros que consultamos en algunas bibliotecas europeas y otras que no hemos tenido a mano y que están citadas en algunos de aquellos libros, como son las listas bibliográficas mencionadas. Además, no todas las obras citadas tratan exclusivamente de la teoría de las paralelas o de las geometrías no enclidianas.

Este trabajo estaba comenzando desde hace varios años; diversas circunstancias habian impedido concluírlo. Esperamos la indulgencia del lector y deseamos que encuentre interesante un conjunto de datos que se hallan diseminados en multitud de obras, muchas de ellas difíciles hoy día de conseguir. Caracas, marzo de 1945.

(*) V. L'Enseignement Mathématique, 1929, p. 349. 


\section{SOBRE LAS GEOMETRIAS NO-EUCLIDIANAS}

Los postulados sobre los cuales se funda la Geometría elemental, enunciados explícitamente en los célebres Elementos de Euclides [10], 2, fueron considerados durante largo tiempo como verdades evidentes de una manera absoluta. Sinembargo, como lo hace notar Mac Leod [134], 29, el gran número de ensayos de demostración del célebre postulado de las paralelas desde la antigüedad hasta la primera mitad del siglo XIX, prueba que ese postulado pareció a los matemáticos menos evidente que los otros.

Todos los ensayos de demostración, algunos de los cuales exponemos en esta Nota, fracasaron, porque en ellos se sustituía implícitamente la propo. sición que se pretendía probar por otra equivalente. Hoy día el asunto está completamente aclarado y la demostración del postulado de Euclides pertenece a la misma categoría que la resolución de los problemas de la cuadratura del círculo, de la trisección del ángulo o de la duplicación del cubo, problemas en los cuales solo se ocupan, como decía Lacaille, los que no son matemáticos.

Desde Euclides hasta Legendre, dice Barbarin [146], 6, es decir durante más de dos mil años, los geómetras desconocieron la verdadera naturaleza del postulado de Euclides y supusieron erróneamente que esta proposición estaba contenida en la noción clásica de la línea recta.

Sin embargo, Lambert (1728-1777) y Taurinus (1794-1874), a pesar de estar convencidos de la verdad del postulado de Euclides, trataron de averiguar las consecuencias que resultarían de negar su validez y llegaron a darse cuenta de que la negación de tal proposición no podía a priori conducir a contradicción lógica por la analogía existente entre las propiedades de las rectas del plano y las de los círculos máximos de la esfera, exceptuando el $6^{\circ}$ postulado: Dos rectas no pueden encerrar un espacio.

Taurinus hizo en 1826 la audaz y profética hipótesis de que existen probablemente superficies curvas en las cuales ciertas curvas contenidas en ellas tienen propiedades análogas a las de las rectas del plano, con excepción del $5^{\circ}$ postulado: Dos rectas de un plano que hacen de un mismo lado, con una tercera, ángulos cuya suma es inferior a dos ángulos rectos se encuentran de ese lado. [146], 7. Veremos más adelante en la interpretación dada en 1868 por Beltrami la exactitud de la hipótesis de Taurinus. Taurinus [127], 67, dedujo las fórmulas de la Trigonometría no euclidiana de las de la Geometría esférica, sustituyendo el radio real $k$ por el radio imaginario $i k$.

Antes de Lambert y de Taurinus, se puede considerar como un precursor de las ideas no euclidianas al italiano Gerolamo Saccheri. En efecto, Saccheri consideró un cuadrilátero birrectángulo en el cual los lados perpendiculares a la base son iguales y, por consiguiente, los otros dos ángulos son iguales; examinó las tres hipótesis relativas a estos ángu- los, a saber: que fueran rectos, agudos u obtusos. Debido a los prejuicios de su época - dice Veronesse [57]- en que se consideraba la Geometría euclidiana como la única posible, Saccheri rechazó la hipótesis que lo habría conducido a la creación de las geometrías no euclidianas, por razones sin fundamento sólido, como veremos luego.

Fue solamente en 1829 cuando aparecieron en ruso, en el Boletín de la Universidad de Kasan, los primeros trabajos de Lobatchewsky (1793-1856) sobre la Geometría no euclidiana que él llamó Geometría imaginaria y después Pangeometría.

Tres años más tarde, en 1832, el geómetra húngaro Juan Bolyai (1802-1860), hijo de Wolfgang Bolyai (1775-1856), publicó en latín un sistema de geometría enteramente semejante al de Lobatche$w s k y$, en un Apéndice a una obra de su padre. La traducción francesa de este Apéndice figura con el $\mathrm{N}^{\circ} 22$ en la Bibliografía que acompaña este trabajo.

La semejanza de las obras de Lobatchewsky y de Bolyai es tan grande que parece increíble, dice Russell [85], 15, que fueran independientes. Se sabe, sin embargo, que Bolyai ignoraba los escritos de Lobatchewsky que solo fueron traducidos en francés en 1837: Géométrie imaginaire (Journal de Crelle, t. XVII) y en alemán en 1840: Geometrische Untersuchungen zur Theorie der Parallellinien.

El gran geómetra $C$. F. Gauss (1777-1855) pasó casi toda su vida, desde la edad de 15 años, reflexionando sobre los fundamentos de la Geometría. El ilustre geómetra comprendió que el "axioma" de las paralelas no podía deducirse de los otros postulados y llegó a fundar un sistema completo de geometría en el cual se supone falso el postulado de Euclides. Llegó así a los mismos resultados que Lobatchewsky y Bolyai como consta de su correspondencia con Schumacher y Wolfgang Bolyai [17], [65], [160]. Sin embargo, Gauss no quiso publicar sus profundas investigaciones, temiendo, como él decía, "los clamores de los beocios" ("Geschrei der Böotier") [111], 73; [160], 11; [127], 57.

Gauss fue no solamente el iniciador de la Geometría no euclidiana, sino que también la influencia de la grande antoridad de semejante maestro fue decisiva en la aceptación de la nueva doctrina. El verdadero triunfo de la Geometría no euclidiana data, dice Bonola [133], 282, del día en que se supo que Gauss estaba convencido de su validez lógica y de la posibilidad de un espacio físico que respondiese a ella.

La hipótesis del ángulo agudo de Saccheri corres ponde a la Geometría de Lobatchewsky. A Riemann (1826-1866), ilustre matemático alemán, se debe la geometría correspondiente al caso del ángulo obtuso.

En la célebre Memoria presentada por Riemann a la Facultad de Filosofía de Gotinga en 1854 (") y publicada en 1867 [70], 280, el autor define la noción de curvatura del espacio en un punto, gene-

(*) Ueber die Hypothesen, welche der Geometrie zu Grunde liegen. 
ralizando la noción de curvatura de un superficie dada por Gauss ("), aplicándola al caso de una multiplicidad de $n$ dimensiones en un espacio de $n+1$ dimensiones.

Riemann toma como elemento infinitesimal del cuadrado de la distancia una forma cuadrática de las diferenciales de las variables, cuyos coeficientes $a_{\mathrm{ij}}$ son funciones de estas variables:

$$
d s^{\imath}=\sum_{i, j} a_{i j} d x_{1} d x_{j} \quad(i, j=1,2 \ldots, n) .
$$

Riemann hace notar que el carácter común de las variedades en que la curvatura es constante en todos los puntos, puede expresarse diciendo que las figuras pueden moverse libremente en ellas sin sufrir deformaciones. Es evidente, en efecto, que las figuras no podrían sufrir traslaciones o rotaciones arbitrarias si la medida de la curvatura en la variedad correspondiente no fuera la misma en todos sus puntos y en todas las direcciones.

Si se designa por $k$ la curvatura del espacio, Riemann halla para el elemento de distancia la fórmula :

$$
d s=\frac{1}{1+\frac{k}{4} \Sigma x_{1}^{2}} \sqrt{\sum d x_{1}^{2}}
$$

en la cual el índice $i$ debe tomar los valores $1,2,3$ para el espacio ordinario.

Según el valor de $k$ se obtienen las diferentes especies de geometrías. A $k=0$ corresponde la Geometría euclidiana o Geometría parabólica; a $k<0$ la Geometría de Lobatchewsky y de Bolyai - Geometría hiperbólica y a $k>0$ la geometría de Riemann o Geometría elíptica. Se supone que en todo el espacio se verifica el principio de superposición de las figuras y que la recta es determinada siempre por dos puntos.

La única superficie de curvatura constante positiva en la Geometría euclidiana es la esfera (**). Se puede interpretar la Geometría elíptica del plano, como lo dijo Lambert, como métrica de la esfera euclidiana, pero no de una manera completa, pues en la esfera dos círculos máximos se cortan siempre en dos puntos opuestos. Para que la recta esté determinada por dos puntos es preciso considerar solamente una región limitada de la superficie llamada región normal.

Tampoco existe, como lo ha demostrado Hilbert [137], 232, ninguna superficie de curvatura constante negativa, sin puntos singulares, sobre la cual sea válida integralmente la Geometría hiperbólica del plano. [146], 36 .

Helmholtz (1821-1894) hizo ver que la forma dada por Riemann al elemento $d s$ para los espacios cuya curvatura es constante, es la úmica compatible con el transporte, sin deformación, de las figuras en el espacio.

(*) Recherches générales sur les surfaces courbes. Trad. M. I. Roger, Grenoble, 1870. p. 14-17.

$(* *)$ H. Liebmann, Göttingen Nachrichten 1899 p. 44, ha demostrado que para que pueda verificarse en su integridad la Geometría plana elíptica sobre una superficie de curvatura constante positiva, esta superficie ha de ser cerrada.
El matemático sueco S. Lie (1842-1899) aplicó a las investigaciones de Riemann y de Helmholtz la teoría de los grupos continuos de transformaciones. Sus trabajos con los de Klein (1849-1925) y de H. Poincaré (1854-1912), han dado a las geometrías no-enclidianas su verdadera significación.

Para terminar estas notas históricas sobre las geometrías no euclidianas, nos resta hablar de la Geometría de Cayley.

El geómetra inglés Cayley (1821-1895) llama absoluto una cónica o una cuádrica con respecto a las cuales se estudian respectivamente las propiedades de las figuras del plano y las de las figuras del espacio, subordinando la Geometría métrica a la proyectiva.

En esta geometría se define el ángulo de dos planos $P_{1} P_{2}$ que se cortan, del modo siguiente: sean

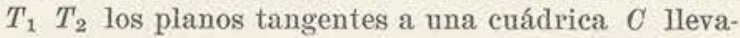
dos por la recta de intersección de $P_{1} P_{2}$; el ángulo de los dos planos se define por la fórmula

$$
V=\frac{1}{2 i} \log \left(P_{1}, P_{2}, T_{1}, T_{2}\right)
$$

siendo el paréntesis la relación anarmónica de los cuatro planos. Cuando esta relación es igual a -1 será $V=\frac{\pi}{2}$ y $\operatorname{los}$ planos $P_{1}, P_{2}$ son conjugados en la cuádrica.

De una manera análoga se define el ángulo de dos rectas que se cortan por medio de las tangentes al absoluto situadas en el mismo plano de las rectas y llevadas desde su punto de corte.

La distancia de dos puntos $M_{1} M_{2}$ se define por la ecuación

$$
M_{1} M_{2}=\frac{k}{2 i} \log \left(M_{1}, M_{2}, A_{1}, A_{2}\right)
$$

siendo $A_{1} A_{2}$ los puntos en que la recta $M_{1} M_{2}$ encuentra el absoluto.

Estas definiciones no se alteran cuando se efectúa una transformación homográfica. Cuando la transformación es por polares recíprocas, los ángulos se cambian en distancias y éstas en ángulos. Ellas concuerdan con las definiciones habituales cuando la cuádrica considerada $C$ se confunde con el círculo del infinito.

Si el absoluto es una cuádrica real convexa, toda recta situada en el interior de la superficie encuentra ésta en dos puntos. La relación anarmónica es siempre positiva y la distancia $M_{1} M_{2}$ es real si se toma la constante $k=R i$, siendo $R$ real.

Considerando únicamente el espacio interior a la superficie, la geometría que resulta de estas definiciones es la de Lobatchewsky y de Bolyai.

Si el absoluto es una cuádrica imaginaria que contiene todos los puntos reales, se halla la Geometría de Riemann.

Cayley había estudiado la métrica proyectiva sin ocuparse de sus relaciones con las geometrías no euclidianas. [43], t. II. p. 561. Fue Félix Klein quien descubrió estas relaciones [26], Math. Ann. t. IV. p. 573; [133], 303. Klein demostró que en la hipótesis no euclídea es posible constituír la Geo- 
metría proyectiva y subordinar, como en el caso de la Geometría euclidiana, las propiedades métricas a las proyectivas.

En cuanto a las representaciones de las geometrías no euclidianas y las pruebas de la indemostrabilidad del postulado de Euclides, entre otras las

\section{DEMOSTRACIONES DEL} DE EUCLIDES DE PROCLUS

Se funda en este postulado: la distancia entre tamente grande cuando se las prolonga suficientemente, en cambio la distancia entre dos paralelas se matiene finita. De aquí deduce que por un punto dado pasa una sola paralela a una recta $\mathrm{y}$ por consiguiente es cierto el $5^{\circ}$ postulado de Euclides.

II.-ENSAYO DE DEMOSTRACION DE NASSIR-EDDIN

Se funda en esta proposición: si las rectas $A D$ y $B C$ (fig. 1) son, la primera perpendicular y la segunda oblicua al segmento $A B$, los segmentos de perpendicular trazada de un punto de $A D$ a la recta $B C$ son menores que $A B$ en la región $D A B C$ en que $B C$ forma ángulo agudo con $A B$ y mayo-

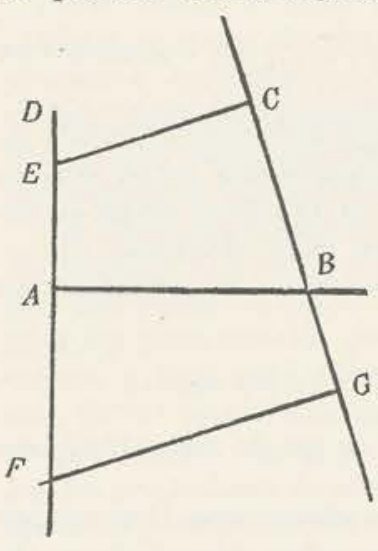

Fig. 1 res que $A B$ en la región $F A B G$ en que $B C$ forma ángulo obtuso con $A B$. De aquí deduce que si dos segmentos perpendiculares a $A B$ son iguales, serán también perpendiculares a $A D$ y la figura formada por las cuatro rectas será un cuadrilátero con sus cuatro ángulos rectos, es decir un rectángulo. Trazando una diagonal se deduce que la suma de los ángulos de un ser la mitad del rectángulo. Como un triángulo cualquiera se puede dividir en dos triángulos rectángulos, resulta que la suma de los ángulos de un triángulo cualquiera es igual a dos ángulos rectos postulado de Euclides.

III--ENSAYO DE DEMOSTRACION DE WALLIS

John Wallis (1616-1703) sustituye el $5^{\circ}$ postulado por la hipótesis de que sea posible construír un bitraria, por analogía con el caso del círculo y el $3^{\text {er }}$ postulado de Euclides: desde cualquier centro $y$ con cualquier radio se puede trazar un círculo.

Ahora, la semejanza de las figuras solo existe en la Geometría euclidiana. [133], 262.

IV.-ENSAYO DE DEMOSTRACION DE SACCHERI

Gerolamo Saccheri (1667-1733) en su obra Euclides ab omni naevo vindicatus; sive geometricus quo stabiliuntur prima ipsa geometriae principia, Mi-
I.-ENSAyo de demostracion Del postulado dos rectas concurrentes crece hasta hacerse infinitriángulo rectángulo es igual a dos rectos, por y de esta última proposición puede deducirse el triángulo semejante a otro dado y de magnitud ar- suministradas por las métricas proyectivas de Cay. ley y de Klein, podrá verlas el lector en [26], [116], [133], [147], [162], etc., no siendo nuestro objeto hacer una exposición completa del asunto.

Pasaremos, pues, a exponer varios ensayos de demostración del célebre postulado de Euclides.

\section{POSTULADO DE EUCLIDES}

lán, 1733, considera un cuadrilátero cuyos lados opuestos $A D$ y $B C$ (fig. 2) son iguales y perpendiculares a la recta $A B$ y examina las tres hipótesis que se presentan como posibles sobre los ángulos iguales $C$ y $D$ del cuadrilátero, a saber: los dos ángulos son rectos; ambos obtusos o ambos agudos. En el primer caso la suma de los tres ángulos de un triángulo es igual a dos rectos de donde deduce Saccheri la validez del $5^{\circ}$ postulado.

En el segundo caso la $D$ suma de los ángulos de un triángulo es superior a dos ángulos rectos. Fundándose en la hipótesis de que la recta es infinita demues- $A$ tra también en el caso del

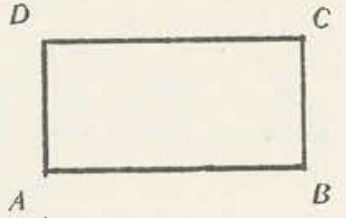

Fig. 2 ángulo obtuso la validez del $5^{\circ}$ postulado. Pero como de este postulado se deduce que la suma de los ángulos de un triángulo es igual a dos ángulos rectos, debe rechazarse la hipótesis del ángulo obtuso. Es decir, esta hipótesis es incompatible con el $6^{\circ}$ postulado.

En el tercer caso la suma de los ángulos de un triángulo es inferior a dos ángulos rectos. Saccheri rechaza también esta hipótesis fundándose en que si ella fuera verdadera, "dos rectas podrían tener una perpendicular común en un punto común situado en el infinito, lo que repugna a la naturaleza de la línea recta". [146], 21.

Siendo pues solamente posible la primera hipótesis, Saccheri creyó así haber demostrado el Postulado de Euclides.

$$
\text { V.-TRABAJOS DE LAMBERT }
$$

El geómetra suizo J.H. Lambert (1728-1777) en su obra Theorie der Parallellinien publicada, después de su muerte, 1786 , considera un cuadrilátero trirrectángulo $\mathrm{y}$ hace las tres hipótesis sobre la naturaleza del cuarto ángulo. Emite la idea de que la hipótesis del ángulo agudo podría ser realizada sobre una cierta superficie que él llama esfera imaginaria. Rechaza la hipótesis del ángulo obtuso porque ella tiene como consecuencia la adopción de una unidad absoluta de longitud.

$$
\begin{aligned}
& \text { VI.- ENSAYO DE DEMOSTRACION DEL POSTULADO } \\
& \text { DE EUCLIDES DE BERTRAND (DE GINEBRA) }
\end{aligned}
$$

Luis Bertrand (1731-1812) ensayó esablecer la teoría de las paralelas sin basarse en ningún postulado para lo cual empleó un procedimiento de bandas infinitas, propuesto en 1667 por Antonio Arnauld, llamado el gran Arnauld (1612-1694). Establecí previamente los lemas siguientes:

$1^{\circ}$ ) Sobre uno de los lados $A X$ (fig. 3 ) de un ángulo recto $X A A^{\prime}$, se toman longitudes iguales 
$A B, B C, C D, \ldots ;$ por los puntos de división se llevan las perpendiculares $B B^{\prime}, C C^{\prime}, D D^{\prime} \ldots$ al lado $A X$. Las bandas $A^{\prime} A B B^{\prime}$ así formadas son ilimitadas en el sentido $A A^{\prime}$; sin embargo, es imposible llenar el espacio angular $X A A^{\prime}$ por grande que sea el número de bandas que se tomen.

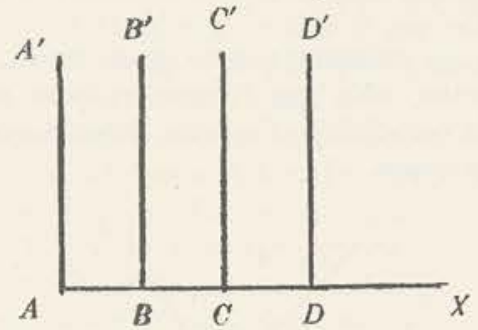

Fig. 3

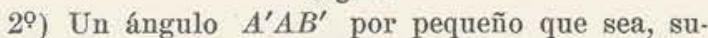
mado sucesivamente a sí mismo puede cubrir completamente el ángulo recto $X A A^{\prime}$.

De estas proposiciones deduce Bertrand que dos rectas, una de ellas perpendicular y la otra oblicua a una tercera son necesariamente concurrentes.

El método es ingenioso y original, pero indudablemente carente en absoluto de rigor matemático pues se funda en la comparación de espacios infinitos. [84], 88 .

\section{VII.-EXPOSICION DEL ENSAYO DE BERTRAND DF DEMOSTRACION DEL POSTULADO DE EUCLIDES Por el Prof. Alberto Lista y Aragón.}

El Prof. Alberto Lista y Aragón, literato y matemático español (1775-1848) publicó un Tratado de Matemáticas puras y mixtas. En el Tratado de Geometría se halla la siguiente exposición de Bertrand para demostrar el postulado de Euclides:

"Sea $E F$ (fig. 4) una recta dada; por el punto cualquiera $C$ tiro a ésta la perpendicular $C D$. Levanto en $C$ la $C B$ perpendicular a $C D$. Las rectas $C B$ y $E F$ serán paralelas por ser perpendiculares a $C D$. Digo que la recta $C A$ que forma ángulo agudo con la $C D$ se ha de encontrar con $E F$. Sea $n$ la relación entre el ángulo recto y el ángulo agudo $B C A$; suponemos que $n$ es un número entero. Tomo sobre $C D \quad n$ número de partes iguales a $C E$ (a partir de $C$ ) y por los puntos de división tiro $G H, M N \ldots$ perpendiculares a $C D$. Se forman así $n$ bandas iguales $B C E F, F E G H, H G M N, \ldots$

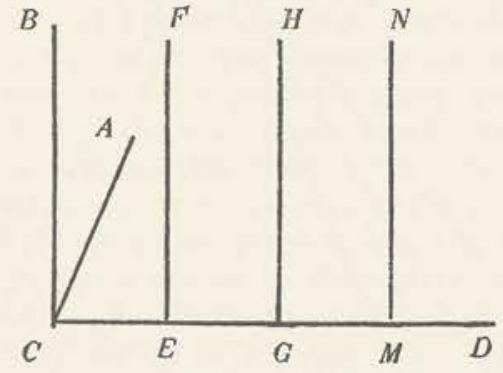

Fig. 4

Ahora, espacio indefinido $B C D>$ espacio indefinido $B C M N$ porque el primer espacio se extiende por la derecha y por la parte superior, mientras que el segundo se extiende sólo por la parte superior. Dividiendo ambos miembros por $n$ resulta:

$$
\text { espacio } B C A>\text { espacio } B C E F
$$

Pero esto es imposible si la recta $C A$ no corta la $E F$. Luego: por el punto $C$ no se puede tirar la $E F$ sino la paralela $C B$.

Si el ángulo $B C A$ no se contiene exactamente en el recto, de modo que $n=m+$ una fracción, tomo una banda más de las que indica el número entero $m$. Siempre será el ángulo recto mayor que la banda total; luego dividiendo el primero por $n$ y la segunda por $m+1>n$ el cociente primero que es $B C A$ será mayor que el segundo, que es $B C E F$ y la conclusión es la misma". [41], 18.

La exposición precedente permite darse cuenta con más claridad de la idea que sirve de base al ensayo de demostración de Bertrand.

$$
\begin{aligned}
& \text { VIII.-ENSAYO DE DEMOSTRACION DEL } 59 \\
& \text { POSTULADO DE EUCLIDES } \\
& \text { Por Schumacher. [17], } 35-36
\end{aligned}
$$

Carta de Schumacher a Gauss.-Me tomo la libertad de someter a su juicio una tentativa que he hecho para demostrar, sin el recurso de las paralelas ni de ninguna teoría la proposición: la suma de los tres ángulos de un triángulo es igual a $180^{\circ}$ de donde se deduciría entonces la demostración del axioma de Euclides. Los únicos principios que supongo establecidos son que la suma de todos los ángulos formados alrededor de un punto es igual a $360^{\circ}$ o a 4 ángulos rectos, y que los ángulos opuestos por el vértice son iguales.

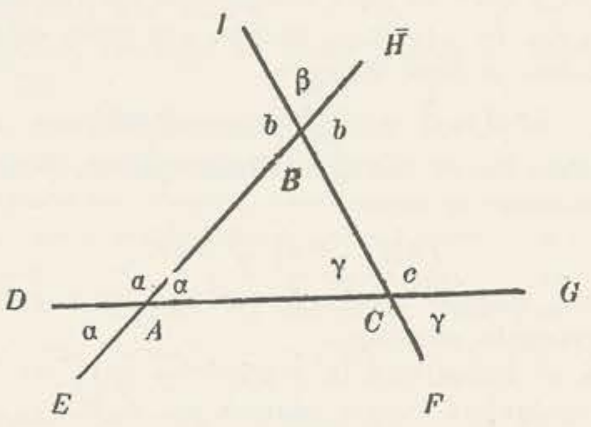

Fig. 5

Prolonguemos indefinidamente los lados de un triángulo rectilíneo $A B C$ (fig. 5) 0 , en otros términos, consideremos un sistema de tres rectas en un plano, formando por sus intersecciones un triángulo $A B C$. Se tiene, para los tres vértices, las ecuaciones

de donde

$$
\begin{aligned}
& 2 a+2 \alpha=4 \text { rectos } \\
& 2 b+2 \beta=4 \quad ", \\
& 2 c+2 \gamma=4 \quad ",
\end{aligned}
$$

$$
\alpha+\beta+\gamma=6 \text { rectos }-(a+b+c)
$$

Esas relaciones subsisten de cualquier manera que estén situados los puntos $A, B, C$, 0 , lo que equi. vale a lo mismo, de cualquier manera que las tres rectas estén trazadas en el plano; dejemos, pues, 
inmóviles las líneas $D G, E H$ y hagamos pasar $I F$ por el punto $A$ (fig. 6) de manera que ella haga con $E H$ el mismo ángulo que en su posición primi-

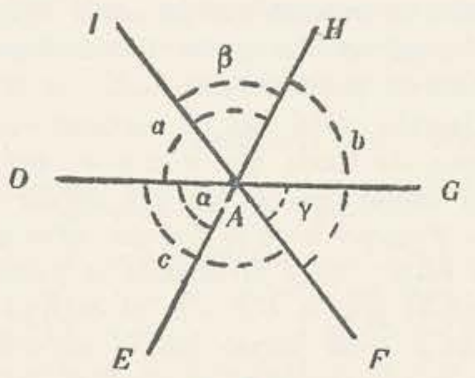

Fig. 6

tiva, o más generalmente, puesto que este ángulo es arbitrario, de modo que ella caiga siempre en el interior del ángulo $a$. Tendremos entonces

$$
\begin{array}{ll}
a+b+c=4 \text { rectos } \\
\text { Luego } & \alpha+\beta+\gamma=2 \text { rectos. }
\end{array}
$$

Se podría objetar a esto que se tiene efectivamente por hipótesis

$$
\begin{aligned}
& b \text { (fig. 5) }=b \text { (fig. 6) } \\
& c(\text { fig. } 5)=c(\text { fig. } 6 \text { ) }
\end{aligned}
$$

pero que la igualdad

Me parece que por ser arbitrario el valor dado a los ángulos, esta demostración no es indispensable.

Tales son los principios de la demostración sobre la cual espero vuestro juicio. Agregaré únicamente para justificar mi razonamiento que es cierto que la segunda operación hace desaparecer el triángulo $A B C$; pero no hace desaparecer los ángulos del triángulo. De cualquier manera que estén situadas las líneas, se tiene siempre

$$
I B H=\beta \quad G C F=\gamma \quad D A E=\alpha
$$

lo mismo en el triángulo finito que en triángulo evanescente; la suma

$$
I A H+G A F+D A E
$$

es, pues, siempre igual a la suma de los ángulos de un triángulo rectilíneo.

Así, se demostrará la proposición para un triángulo cualquiera (cuyos ángulos son $A, B, C$ ), tirando las líneas $D G \quad E H$ de manera que se tenga $\alpha=A$ y haciendo además $I A H=B \quad G A F=C$.

Si entonces $I A F$ no fuera una línea recta sino una línea quebrada $I A F^{\prime}$, el ángulo $C$ se hallaría, es cierto, más pequeño de $d c$; pero el ángulo $b$ sería mayor en la misma cantidad y, por consiguiente, la suma de esos ángulos no habría cambiado, y tendríamos, lo que nos es necesario para la demostración, la igualdad

$$
b+c \text { (fig. } 5)=b+c(\text { fig. } 6)
$$

Copenhague, 3 de mayo de 1831 .

Respuesta de Gauss. [17], 37.

Examinado bien lo que usted me escribe respecto de las paralelas, ha empleado en sus silogismos, sin enunciarla explícitamente, una proposición que puede formularse así:
Si dos rectas que se cortan, (1) y (2), (fig. 7) hacen respectivamente, con una tercera recta (3) que las encuentra, los ángulos $A^{\prime}, A^{\prime \prime}$ y que una cuarta recta (4) situada en el mismo plano sea cortada del mismo modo por (1) bajo el ángulo $A^{\prime}$, entonces (4) será cortada por (2) bajo el ángulo $A^{\prime \prime}$.

Ahora, no solamente esta proposición necesita demostración, sino que se puede decir que en el fondo ella constituye el mismo teorema que se trata de demostrar.

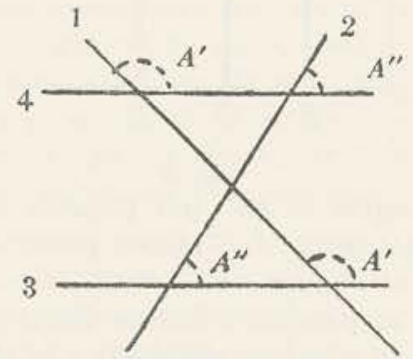

Fig. 7

Desde hace algunas semanas he comenzado a escribir algunos resultados de mis propias meditaciones sobre este asunto, las cuales datan en parte de hace cuarenta años y no había redactado nunca nada, lo que me ha forzado a recomenzar tres o cuatro veces todo el trabajo mentalmente. No quisiera, sinembargo, que todo esto pereciera junto conmigo.

Goettingue, 17 de mayo de 1931.

$$
\begin{aligned}
& \text { IX.-ENSAYO DE DEMOSTRACION DEL } \\
& \text { POSTUKADO DE EUCLIDES } \\
& \text { de J. Richard. [97], 50-52 }
\end{aligned}
$$

Richard formuló una demostración del $5^{\circ}$ postulado modificando una demostración dada por Carton (Comptes Rendus de l'Acad. des Sc. de Paris, 1867).

Se considera una serie de $n$ triángulos (fig. 8) $A B C, C D E, E F G, \ldots H K L, L M N$

todos iguales y cuyas bases están situadas sobre

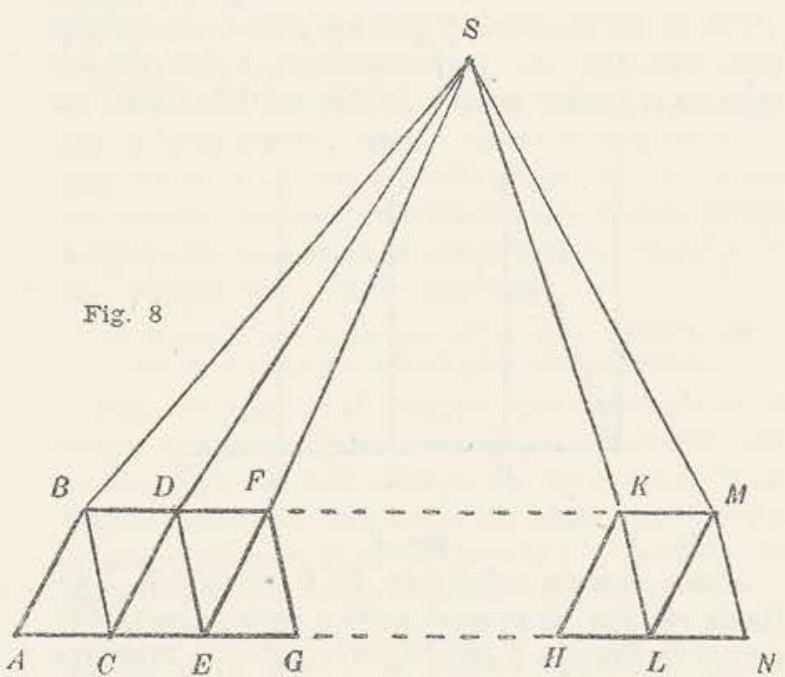


una misma recta $A N$. Uniendo los vértices $B, D, F^{\prime}$, ... $K, M$ de dos en dos por medio de rectas se forma una nueva serie de $n-1$ triángulos iguales entre sí, pero no forzosamente iguales a los primeros (serían iguales a los primeros en Geometría cuclidiana). Se toma un punto $S$ por encima de esta red de triángulos y se une $S$ con los vértices $B, D, F \ldots K, M$. Se forma así $n-1$ nuevos triángulos con el vértice común $S$.

Suponiendo que la suma de los ángulos de un triángulo sea inferior a 2 ángulos rectos, sea $2-a$ la suma de los ángulos de cada triángulo de la $1^{\text {a }}$ serie; $2-b$ la suma de los ángulos de cada triángulo de la $2^{\text {a }}$ serie $; 2-\omega$, siendo $\omega$ variable, la de un triángulo cualquiera de la $3^{\text {a }}$ serie. La suma de todos los ángulos será

$$
\begin{aligned}
& \quad(2-a) n+(2-b)(n-1)+2(n-1)-\Sigma \omega \\
& \text { o sea } \quad 6 n-4-n(a+b)+b-\Sigma(1) .
\end{aligned}
$$

Esta suma puede calcularse de otro modo; sea $P$ la suma de los ángulos del pentágono $S B A N M$; en cada uno de los vértices $D, F, \ldots K$ en número de $n-2$ hay 4 ángulos rectos y en cada uno de los puntos $C, E, G, \ldots H, L$ en número de $n-1$ hay 2 ángulos rectos. La suma total será pues:

$$
\begin{aligned}
& P+4(n-2)+2(n-1) \\
& \text { O sea } \quad P+6 n-10 \text {. } \\
& 6 n-4-n(a+b)+b-\Sigma \omega=P+6 n-10 \\
& \text { De donde } \quad P=6-[n a+(n-1) b+\Sigma \omega]
\end{aligned}
$$

Resulta de aquí que, por pequeños que sean $a, b, \Sigma \omega$ se podrá siempre escoger $n$ suficientemente grande para que $P$ sea negativo, lo que es absurdo. Por consiguiente la suma de los ángulos de un triángulo no puede ser inferior a 2 ángulos rectos.

Pero esta conclusión depende de la hipótesis implícita siguiente: Se ha supuesto que, por lejos que se prolongue la construcción de los triángulos se podrá siempre hallar un punto $S$ por encima de la primera recta $B D$ y por encima de la ultima $K M$, lo cual no es evidente a priori. Es decir, se supone que la construcción es siempre posible por grande que se suponga $n$. Esto se verifica en la hipótesis euclidiana y se reemplaza así en esta demostración el postulado de Euclides por otro postulado.

Esta demostración tiene analogía con una falsa demostración en que se pretende probar que un ángulo recto es igual a un ángulo agudo. La demostración es irreprochable desde el punto de vista de los razonamientos, pero se funda en una hipótesis falsa: se supone que dos rectas se cortan de un cierto lado de la figura cuando en realidad se cortan del lado opuesto. (V. Lietzmann and Trier, Wo Steckt der Fehler? Leipzig, 1917, p. 22).

Algo análogo ocurriría en la Geometría de Lobatchevsky con la construcción de la demostración precedente.

NoтA. - Richard al formular esta demostración sabía dónde se encuentra el error de la misma.
X.- DEMOSTRACION DEL TEOREMA SOBRE LA SUMA DE LOS TRES ANGULOS DE UN TRIANGULO

Por Adrien Marie Legendre

El ilustre matemático francés Legendre (17521833) se ocupó mucho de la teoría de las paralelas y ensayó vanamente demostrar el $5^{\circ}$ postulado de Enclides.

Sin embargo, sus trabajos sobre este asunto son muy notables y no contienen los errores groseros de que adolecen otras pseudodemostraciones del célebre postulado.

Legendre demostró estos teoremas: $1^{\circ}$ ) En un triángulo rectilíneo la suma de sus ángulos no puede ser superior a dos ángulos rectos. $2^{\circ}$ ) Si en un triángulo rectilíneo la suma de sus ángulos es igual a dos rectos, será lo mismo en todos los triángulos. El primero de estos teoremas se halla en la $12^{\text {a }}$ edición de sus célebres Eléments de Géométrie, París, 1823 (prop. XIX del Libro I, pág. 20). [11], [17], 7. El segundo fue publicado solamente en 1833.

La demostración del $5^{\circ}$ postulado de Euclides a la que vamos a referirnos, es una demostración indirecta. Legendre prueba que la suma de los tres ángulos de un triángulo rectilíneo es igual a dos ángulos rectos, sin valerse de la teoría de las paralelas. Ahora, el postulado de Euclides es una consecuencia como se sabe del teorema sobre la suma de los tres ángulos de un triángulo. Sin embargo, aun cuando la demostración de Legendre no contiene petición de principio, no es una prueba del postulado pues reemplaza éste por el postulado de la homogeneidad de las fórmulas de la Geometría, homogeneidad que sólo se verifica en la Geometría euclidiana. He aquí la demostración de Legendre. [11], 181:

"Se demuestra inmediatamente por la superposición y sin ninguna proposición preliminar que dos triángulos son iguales, cuando tienen un lado igual adyacente a dos ángulos iguales. Llamemos $p$ el lado de que se trata, $A$ y $B$ los dos ángulos adyacentes, $C$ el tercer ángulo. Es necesario, pues, que el ángulo $C$ sea enteramente determinado cuando se conocen los ángulos $A$ y $B$ y el lado $p$; pues si varios ángulos $C$ pudiesen corresponder a los tres datos $A, B, p$ habría otros tantos triángulos diferentes que tendrían un lado igual adyacente a dos ángulos iguales, lo que es imposible: luego el ángulo $C$ debe ser una función determinada de las tres cantidades $A, B, p$ lo que expreso así:

$$
C=\varphi(A, B, p)
$$

Sea el ángulo recto igual a la unidad; entonces los ángulos $A, B, C$ serán números comprendidos entre 0 y 2 y puesto que $C=\varphi(A, B, p)$ digo que la línea $p$ no debe entrar en la función $\varphi$. En efecto, se ha visto que $C$ debe ser enteramente determinado por los solos datos $A, B, p$, sin ningún otro ángulo o línea; pero la línea $p$ es heterogénea con los números $A, B, C$; y si se tuviera una ecuación cualquiera entre $A, B, C, p$, se podría deducir el valor de $p$ en función de $A, B, C$; de donde resultaría que $p$ es igual a un número, lo que es absur- 
do: luego $p$ no puede entrar en la función $\varphi$ y se tiene simplemente $C=\varphi(A, B)$.

Esta formula prueba ya que, si dos ángulos de un triángulo son iguales a dos ángulos de otro triángulo, los terceros deben ser iguales y sentado esto es fácil llegar al teorema que deseamos demostrar $\left(^{*}\right)$.

Sea en primer lugar $A B C$ (fig. 9) un triángulo rectángulo en $A$; del punto $A$ bájese $A D$ perpendicular sobre la hipotenusa. Los ángulos $B$ y $D$ del triángulo $A B D$ son iguales a los ángulos $B$ y $A$ del triángulo $B A C$; luego, según lo que se acaba de demostrar, el tercero $B A D$ es igual al tercero $C$. Por la misma razón el ángulo $D A C=B$, luego $B A D+D A C$ (o $B A C)=B+C$. Ahora, el ángulo $B A C$ es recto; luego: la suma de los dos ángulos agudos de un triángulo rectángulo vale un ángulo recto.

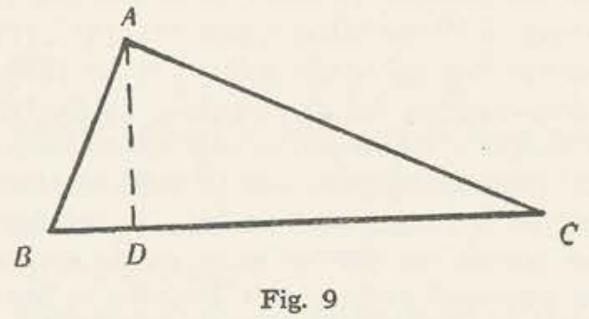

Sea ahora $B A C$ (fig. 10) un triángulo cualquiera y $B C$ un lado que no sea menor que cada uno de los otros dos. Si del ángulo opuesto $A$ se baja la perpendicular $A D$ sobre $B C$, esta perpendicular caerá en el interior del triángulo $A B C$ y lo dividirá en dos triángulos rectángulos $B A D, D A C$. Ahora, en el triángulo rectángulo $B A D$ los dos ángulos $B A D, A B D$ sumados valen un ángulo recto; en el triángulo rectángulo $D A C$ los dos ángulos $D A C, A C D$ sumados valen también un án-

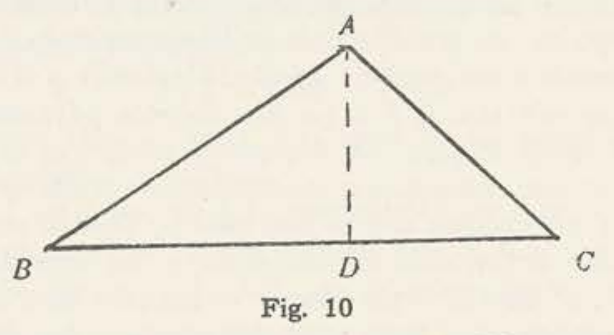

gulo recto. Luego los cuatro reunidos o, solamente los tres $B A C, A B C, A C B$ sumados valen dos ángulos rectos. Luego en todo triángulo recti-

(*) Se ha objetado contra esta demostración que, si se la aplicara punto por punto a los tríángulos esféricos resultaría que conocidos dos ángulos se podría determinar el tercero, lo que no sucede en esta clase de triángulos. La respuesta es, que en los triángulos esféricos hay un elemento más que en los triángulos planos y este elemento es el radio de la esfera del cual no se debe hacer abstracción. Sea, pues, $r$ el radio, entonces en rez de ser $\mathrm{C}=\varphi(\mathrm{A}, \mathrm{B}, \mathrm{p})$, se tendrá $\mathrm{C}=\varphi(\mathrm{A}, \mathrm{B}, \mathrm{p}, \mathrm{r})$ o únicamente $\mathrm{C}=\varphi\left(\mathrm{AB}_{\mathrm{r}}^{\mathrm{D}}\right)$, en virtud de la ley de los homogéneos. Ahora, puesto que la relación ${ }_{\mathrm{r}}^{\mathrm{p}}$ es un número, como $\mathrm{A}, \mathrm{B}, \mathrm{C}$ nada impide que ${ }_{\mathrm{r}}^{\mathrm{p}}$ no se halle en la función $\varphi$ y entonces no puede concluírse que $\mathrm{C}=\varphi(\mathrm{A}, \mathrm{B})$. líneo la suma de los tres ángulos es igual a dos án. gulos rectos.

Se ve por esto que este teorema, considerado $a$ priori, no depende de un encadenamiento de proposiciones y que él se deduce inmediatamente del principio de la homogeneidad, principio que debe verificarse en toda relación entre cantidades cualesquiera". [3], 273-282.

$$
\text { *** }
$$

La otra demostración del teorema sobre la suma de los ángulos de un triángulo dado por Legendre, es decir el teorema XIX de sus célebres Eléments de Géométrie, prueba [17], 7, que la suma de los ángulos de un triángulo no puede ser superior a dos ángulos rectos. No se olvide que Legendre supone implícitamente en esta demostración que la recta es infinita y por consiguiente la demostración no es válida en la Geometría de Riemann. [111], 73.

\section{XI.-ENSAYO DE DEMOSTRACION DEL POSTULADO DE EUCLIDES}

Por Adrien Marie Legendre. [11]

Sea $B A C$ un ángulo dado (fig. 11) y $M$ un punto situado en el interior de este ángulo. Sea $A D$ la bișectriz del ángulo $B A C$; sea $M P$ la perpendicular a $A D$ bajada del punto $M$. La recta $M P$ prolongada en los dos sentidos deberá encontrar los lados del ángulo. Pues, a causa de la simetría,

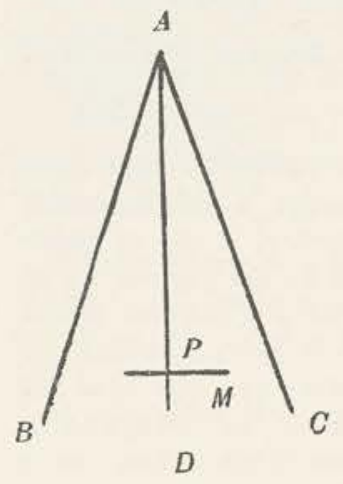

Fig. 11

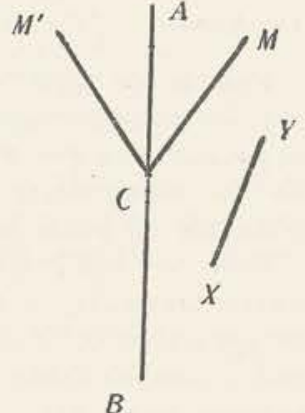

Fig. 12 si encuentra uno de los lados deberá encontrar el otro; si no encuentra uno de los lados, tampoco encontrará el otro. En este último caso la recta estaría toda entera encerrada en el espacio comprendido entre los lados del ángulo $B A C$. Pero esto repugna a la naturaleza de la línea recta. En efecto, toda recta $A B$ (fig. 12) trazada sobre un plano y prolongada indefinidamente divide el plano en dos partes que superpuestas coinciden en toda su extensión y son perfectamente iguales. La parte $A M B$ del plano total, situada de un lado de $A B$ es igual en todo a la parte $A M^{\prime} B$ situada del otro lado; pues si se toma un punto fijo $C$ sobre $A B$, un punto cualquiera $M$ estará determinado por la distancia $C M$ y el ángulo $A C M$. El punto $M^{\prime}$ simétrico de $M$, es decir tal que $C M^{\prime}=C M, A C M^{\prime}=A C M$, se confundirá con $M$ cuando se superpongan las dos par- 
tes en que el plano queda dividido por la recta $A B$.

Supongamos ahora, si es posible, que una recta ilimitada $X Y$ esté encerrada enteramente en un espacio angular cualquiera, por ejemplo, en el ángulo $B C M$; ella dividirá en dos partes iguales o desiguales, la parte del plano comprendido en el ángulo $B C M$. Esta parte tiene su correspondiente $B C M^{\prime}$ situada del otro lado de $B C$; pero como además de esas dos partes iguales del plano, existen otras dos encerradas en los ángulos iguales $A C M, A C M^{\prime}$, se ve que el espacio angular $B C M$ no es la mitad de todo el plano. Luego la recta $X Y$ que se supone divide en dos porciones el espacio $B C M$, no podrá dividir la totalidad del plano sino en dos partes desiguales, lo que es contrario a la naturaleza de la línea recta.

Sentado esto se puede demostrar el postulado de Euclides. Este postulado se reduce fácilmente, como se sabe, al caso en que una de las rectas $A C$ (figura 13), siendo perpendicular a $A B$ la otra $B D$ hace con $A B$ un ángulo $A B D$ menor que un recto. Se trata, pues, de probar que en ese caso $B D$ prolongada debe encontrar $A C$. En efecto, si no fuese así, prolongando $A C$ hacia el lado opuesto $A C^{\prime} \mathrm{y}$ ha-

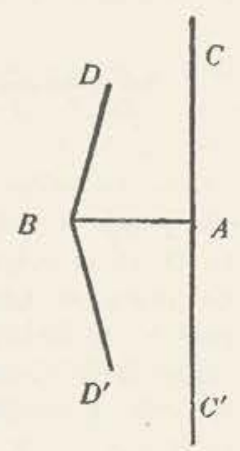

Fig. 13 ciendo el ángulo $A B D^{\prime}=A B D$, la recta $C C^{\prime}$ estaría comprendida toda entera en el ángulo $D B D^{\prime}$ menor que dos rectos, lo que es imposible [11], 279-280.

En esta demostración se reemplaza el $5^{\circ}$ postulado por otro equivalente $\mathrm{y}$ adolece además del defecto de comparar entre sí espacios infinitos.

XII.-FALSA DEMOSTRACION DEL TEOREMA SOBRE LA SUMA DE LOS TRES ANGULOS DE UN TRIANGULO

Sea $A B C$ (fig. 14) un triángulo cuyos ángulos son $\alpha, \beta, \gamma$. Se prolonga el lado $A B$ según $B B^{\prime}$, el lado $B C$ según $C C^{\prime}$ y el lado $C A$ en la dirección $A A^{\prime}$. Sea $\alpha^{\prime}, \beta^{\prime}, \gamma^{\prime}$ los ángulos exteriores adyacentes a $\alpha, \beta, \gamma$ respectivamente.

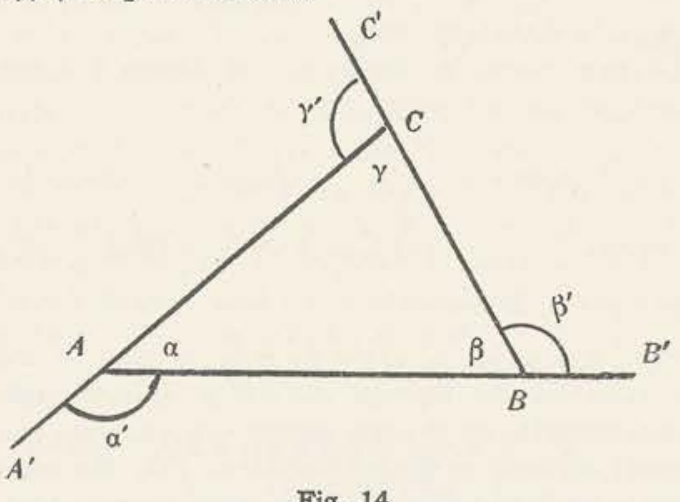

Fig. 14

Se hace girar ahora $B B^{\prime}$ alrededor del punto $B$ del ángulo $\beta^{\prime}$ hasta que $B B^{\prime}$ coincida con $B C$; se hace deslizar $B B^{\prime}$ sobre $B C$ hasta que el punto $B$ coincida con $C$ y $B B^{\prime}$ con $C C^{\prime}$; se hace girar $C C^{\prime}$ del ángulo $\gamma^{\prime}$ alrededor del punto $C$ hasta que $C C^{\prime}$ se confunda con $C A$ y se hace deslizar $C C^{\prime}$ sobre $C A$ hasta que ocupe la posición $A A^{\prime}$. Se hace girar $A A^{\prime}$ alrededor de $A$ del ángulo $\alpha^{\prime}$ de modo de hacerle coincidir con $A B$ y se hace deslizar hasta que llegue a la posición $B B^{\prime}$. Puesto que la recta $B B^{\prime}$ partió de su posición primitiva para volver a ella, se tendrá que la suma de los ángulos de giración es igual a 4 ángulos rectos:

$$
\alpha^{\prime}+\beta^{\prime}+\gamma^{\prime}=4
$$

Pero:

$$
\begin{gathered}
\alpha+\alpha^{\prime}=2 \quad \beta+\beta^{\prime}=2 \quad \gamma+\gamma^{\prime}=2 \\
\alpha+\beta+\gamma=2
\end{gathered}
$$

Luego:

El resultado es cierto en Geometría euclidiana porque trazando por $B$ (fig. 15) la recta $B A^{\prime}$ paralela a $C A$, el ángulo $A^{\prime} B B^{\prime}$ es igual a $\alpha^{\prime}$ y el ángulo $C B A^{\prime}$ es igual a $\gamma^{\prime}$ y la ecuación $\alpha^{\prime}+\beta^{\prime}+\gamma^{\prime}=4$ es entonces cierta.

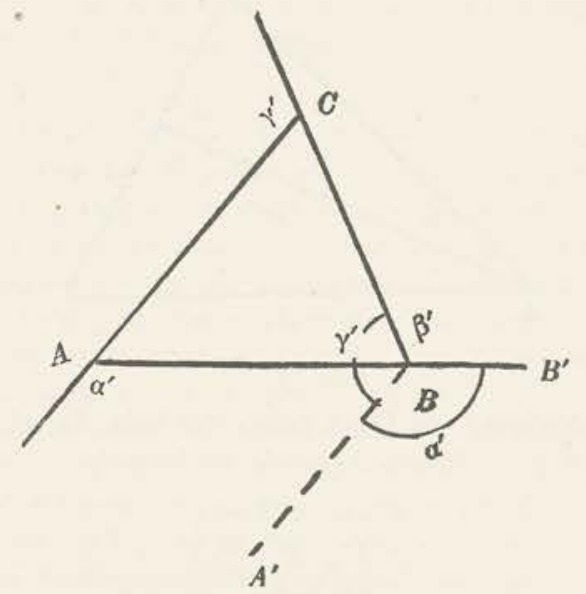

Fig. 15

Pero se ve por esto mismo que la demostración no es independiente del $5^{\circ}$ postulado y que, para que ella sea válida en Geometría euclidiana sería preciso admitir otro postulado, a saber: que la ecuación $\quad \alpha^{\prime}+\beta^{\prime}+\gamma^{\prime}=4 \quad$ sea verificada.

En efecto, se podría aplicar punto por punto el mismo razonamiento a un triángulo esférico (fig. 16), puesto que es posible hacer girar el

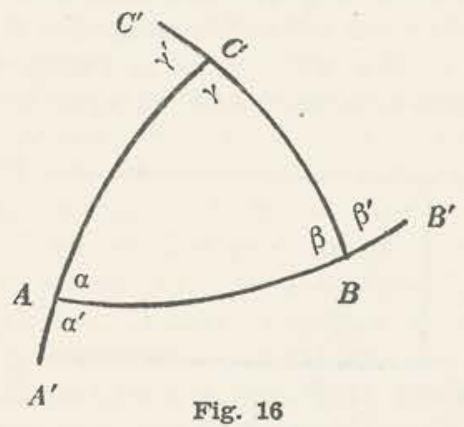

arco de círculo máximo $B B^{\prime}$ hasta que coincida con $B C$ y deslizarlo sobre $B C\left(^{*}\right)$ hasta que coin-

(*) Puesto que todos los círculos máximos de la esfera son iguales y que por todo punto de la superficie de la esfera pasan infinitos círculos máximos. 
cida con $C C^{\prime}$, etc. de donde se concluirá que la suma de los tres ángulos de un triángulo esférico es igual a dos rectos, to que es absurdo. (V. Lietzmann und Trier, Wo Steckt der Fehler? Leipzig, 1917, p. 27).

Esta demostración es uno de los ejemplos más claros de las demostraciones en que se reemplaza el postulado $5^{\circ}$ por otro postulado más difícil de admitir.

Es, en el fondo, el mismo ensayo de demostración de Schumacher ya expuesto.

\section{XIII.-ENSAYO DE DEMOSTRACION DEL} POSTULADO DE EUCLIDES

Si se acepta el siguiente postulado: la suma de los tres ángulos de un triángulo rectilíneo es una cantidad constante $k$, se deducirá el postulado de Euclides. En efecto, sea $A B C$ (fig. 17) un triángu-

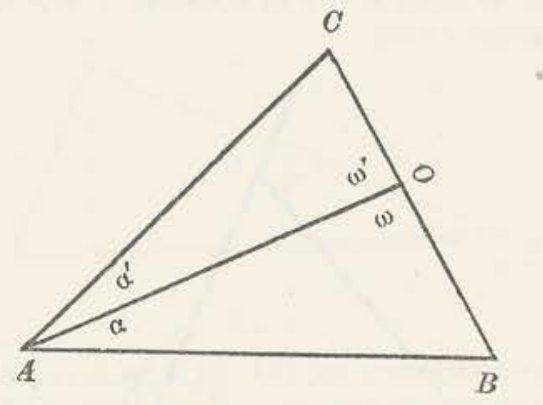

Fig. 17

lo cualquiera; sea $O$ un punto del lado $B C$ situado entre $B$ y $C$; úmase $A$ con $O$. Se tendrá.

$$
\begin{gathered}
\alpha+\omega+B=k \\
\alpha^{\prime}+\omega^{\prime}+C=k \\
k=A+B+C
\end{gathered}
$$

Sumando miembro a miembro, estas ecuaciones, se tendrá:

$$
\omega+\omega^{\prime}=k
$$

y como $\omega+\omega^{\prime}=2$ ángulos rectos, resulta:

$$
k=2 \text { ángulos rectos. }
$$

Luego: la suma de los tres ángulos de un triángulo rectilíneo es igual a dos ángulos rectos.

Sea ahora $P R$ (fig. 18) una recta $y$ un punto fuera de ella; sea $M P$ la perpendicular a $P R$ bajada desde $M$. Sea $M N$ una recta cualquiera. Si el ángulo $P M N$ es inferior a $90^{\circ}$, la recta $M N$ cortará

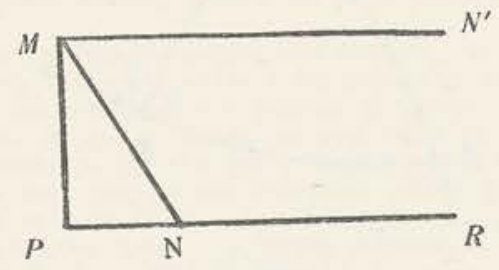

Fig. 18

la $P R$; pero si el ángulo $P M N^{\prime}=90^{\circ}$, la recta $M N^{\prime}$ no cortará la $P R$, pues de lo contrario se tendría un triángulo rectilíneo en el cual la suma de los tres ángulos sería superior a dos ángulos rectos.

\section{XIV-XV.-DNSAYOS DE DEMOSTRACION DEL POSTULADO DE EUCLIDES}

del doctor Julio Garavito Armero (1865-1920)

En 1918 publicó el doctor Garavito, antiguo Director del Observatorio de Bogotá, un estudio intitulado "Nota sobre las Geometrías planas no euclídeas" en el cual inserta dos ensayos de demostración del célebre postulado. [126].

En el primer ensayo, Garavito considera una recta ilimitada $L^{\prime} L$ (fig. 19) y un punto $P$ fuera de

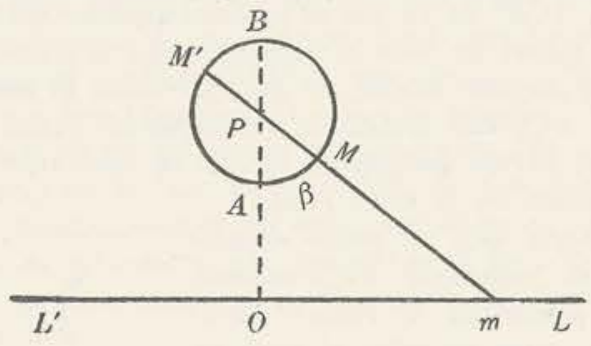

Fig. 19

ella y baja la perpendicular $P O$ sobre $L^{\prime} L$. EI punto $O$ es el origen para determinar la posición de un punto $m$ que se mueve sobre $L^{\prime} L$, y designa por $z$ la distancia $O m$ con las convenciones de signo de la Geometría analítica. Trazando la recta $P m$ y designado por $\beta$ el ángulo $O P M$, resulta que $z$ y tang $\beta$ son cantidades reales, va. rían de $-\infty$ a $+\infty$ y a un valor de $z$ corresponde uno solo de tang $\beta$ y recíprocamente; de modo que $z$ es función uniforme de $\operatorname{tang} \beta$. Garavito concluye que estas dos cantidades están li. gadas por una ecuación de la forma

$$
A \approx \operatorname{tang} \beta+B z+C \operatorname{tang} \beta+D=0 \text {. }
$$

Puesto que si $z=0$ es $\beta=0$ y por tanto tang $\beta=0$, resulta $D=0$. Dando a $\beta$ dos valores iguales y de signos contrarios, los valores correspondientes de $z$ serán iguales y de signos contrarios y se deduce $A=o$. La ecuación se reduce a

$B z+C \operatorname{tang} \beta=0 \quad$ De donde $\quad z=g \operatorname{tang} \beta$ designando por $g$ el cociente $-\frac{B}{C}$, que, como se sabe es la distancia $O P$.

De aquí continúa Garavito: "Si damos a $\beta$ cualquiera de las dos series de valores

$$
\beta_{1}=\frac{\pi}{2}+2 n \pi \quad \text { o } \quad \beta_{2}=\frac{\pi}{2}+(2 n+1) \pi
$$

se tendrá:

$$
\operatorname{tang} \beta_{1}=\operatorname{tang} \beta_{2}=\operatorname{tang} \frac{\pi}{2}=\infty
$$

y por tanto, la ecuación $z=g$ tang $\beta$, dará $z=\infty$ ".

"Las dos series de arcos no definen sino un mismo diámetro del círculo $A M B M^{\prime} A$ (círculo cualquiera descrito de $P$ como centro con un radio cualquiera), el cual es perpendicular a $P O$. En consecuencia no habrá sino una sola recta trazada por $P$ que no corta a $L^{\prime} O L$. Esta recta es la perpendicular a $P O$. Cualquiera otro valor de $\beta$ dará valor finito para tang $\beta$ y por tanto para $\approx$ (Postulado de Euclides)". [126], 9. 
Garavito considera inatacable su razonamiento y, como da por sentado que Lobatchevosky llegó forzosamente a las mismas conclusiones que él, exclama: "Grande ha debido ser la sorpresa de Lobattcheffsky al hallarse, cuando menos lo esperaba, frente a frente con el postulado de Euclides".

¿Dónde se halla la equivocación de Garavito? Es fácil responder:

El error de Garavito al haber creído demostrar el Postulado de Euclides, consiste en no haberse dado cuenta de que reemplazó el célebre postulado por otra proposición no demostrada. En efecto, Garavito supone, implícitamente, que la relación que liga la distancia $z$ y la tangente del ángulo $\beta$ es algebráica, lo cual no es de ningún modo evidente a priori. Ahora, como dice el gran geómetra francés Darboux [125], 6:

Si la relación entre dos cantidades reales $x, y$ es tal que a un valor de la una corresponde un solo valor de la otra y si por la naturaleza de la cuestión se sabe que esta relación es algebraica, se podrá concluír que ella es de la forma

$$
A x y+B x+C y+D=0
$$

siendo $A, B, C, D$ constantes. Pero, si no se sabe a priori que la relación es algebraica, se podrá imaginar una multitud de otras formas de la relación, por ejemplo $\quad y=\varphi(x)$

en que $\varphi$ designa una función que crece de $-\infty$ a $+\infty$ cuando $x$ crece de la misma manera. Por ejemplo, se podrá poner

$$
y=e^{x}-e^{-x} \text {. }
$$

Garavito no justifica su postulado de que la relación que liga un lado del ángulo recto de un triángulo rectángulo y la tangente del ángulo opuesto es algebraica, porque es imposible encontrar a priori fundamento a dicha hipótesis. Ahora, como precisamente en el sistema euclidiano esta relación es algebraica, resulta que haciendo tal hipótesis se cae forzosamente en el postulado de Euclides. La demostración de Garavito no prueba, pnes, nada.

Veamos, en efecto, cómo en las otras geometrías la relación en cuestión no es algebraica.

Si se rechaza el postulado de Euclides y se admiten como verdaderos los otros postulados de la Geometría, Lobatchewsky ha demostrado [17], 33, [fórmula (8) ]; [18], 23 [fórmula (9) ], que en un triángulo rectilíneo cualquiera cuyos lados son $a, b, c$ y sus ángulos opuestos $A, B, C$, se tiene, designando por $\pi(x)$ el ángulo de paralelismo de $x$, es decir $\quad \operatorname{tang} 1 / 2 \pi(x)=e^{-\frac{x}{b}}$. y que:

$$
\operatorname{cotang} B \operatorname{sen} A \operatorname{sen} \pi(c)+\cos A=\frac{\cos \pi(c)}{\cos \pi(b)} .
$$

Suponiendo ahora $A=\frac{\pi}{2}$ se tendrá para el triángulo rectángulo: $\operatorname{tang} B=\operatorname{tang} \pi(c) \cos \pi(b)$.

Esta fórmula general comprende como casos particulares los que corresponden a la Geometría rie- manniana y a la Geometría euclidiana. En efecto, cambiando $k$ por $i R$ se tiene para el triángulo rectángulo esférico:

$$
\operatorname{tang} \frac{b}{R}=\operatorname{tang} B \operatorname{sen} \frac{c}{R} .
$$

Suponiendo $a, b, c$ infinitamente pequeños con relación a $R$ o a $R$ infinitamente grande resulta:

$$
b=\operatorname{cotang} B
$$

fórmula del triángulo rectángulo en Geometría enclidiana.

El caso de ser algebraica la relación entre un lado de un triángulo rectángulo y la tangente del ángulo opuesto es, pues, un caso particular del caso general en el cual dicha relación no es algebraica y ese caso particular corresponde precisamente a la Geometría enclidiana, como ya lo habiamos dicho.

Esta demostración de Garavito es también un claro ejemplo de los ensayos de demostración en que se reemplaza el postulado de Euclides por otro postulado más difícil de admitir.

$$
\because * \text { * }
$$

El segundo ensayo de demostración del postulado de Euclides, expuesto por Garavito, se funda en la interpretación geométrica de las soluciones de los sistemas de ecuaciones lineales con dos y tres incógnitas que enseña la Geometría analítica [105], 5,$6 ;[112]$, 39. Esta interpretación consiste, como se sabe, en convenir a priori en llamar punto a un conjunto de dos o de tres variables (o de $n$ variables en el hiperespacio), recta a una ecuación de primer grado entre dos variables o a un sistema de dos ecuaciones de primer grado entre tres variables, etc. [134], 20-23. Las soluciones de las ecuaciones lineales se pueden enunciar entonces en este lenguaje geométrico convencional y así resulta que el postulado de Euclides corresponde al caso de imposibilidad o incompatibilidad del sistema de dos ecuaciones de primer grado con dos variables. Es decir, el caso en que las soluciones son infinitas, corresponde al caso de la paralela única (Postulado de Euclides). Y concluye Garavito:

"En todo lo que acabamos de decir nos hemos referido al Algebra pura: las variables no son coordenadas sino simples cantidades numéricas y por tanto no es el caso de señalar petición de principio ni cárculo vicioso".

El error de Garavito consiste en haber olvidado que los teoremas conocidos de Geometría, enunciados cuando se consideran los puntos, líneas y superficies como variedades definidas analíticamente no son en realidad verdades geométricas, sino verdades enunciadas en un lenguaje geométrico, las cuales no se aplican a figuras concretas de Geometría mientras no se acuerden previamente con los postulados fundamentales o de base. [83], 189; [112], 43.

Pretender, pues, que la condición de incompatibilidad de ecuaciones de primer grado sea una demostración del célebre Postulado, es caer en círculo vi. cioso o petición de principio. En efecto, para probar que una ecuación de primer grado con dos variables representa una línea recta y que, recíprocamente, 
toda línea recta está representada por una ecuación de primer grado, es preciso basarse en el postulado mismo que se pretende demostrar. Es decir, para acordar en este caso el lenguaje analítico con el geométrico es preciso admitir previamente el postulado de Euclides.

Algo análogo sucede con la teoría de las integrales de variable compleja de Cauchy, como to ha hecho notar Laurent [40], 381. En esta teoría se hace uso del lenguaje geométrico por comodidad de la exposición, lenguaje que es perfectamente correcto aun cuando no tuviéramos ninguna noción del espacio ordinario. Resulta, pues, que si, en apariencia, podría parecer que la teoría de las integrales definidas de Cauchy y las consecuencias que se deducen sobre las propiedades de las funciones, están fundadas en la Geometría, son en realidad absolutamente independientes del Postulado de Euclides, a pesar de que se haya hecho uso de coordenadas. Se puede, en efecto, razonar sin figuras, definiendo aun en Geometría de dos dimensiones, las líneas y las superficies como se hace en el hiperespacio en general.

Sería, pues, grave error creer que el Postulado de Euclides podría demostrarse fundándose en el hecho de que las integrales halladas por medio del cálculo de los residuos pueden determinarse, con los mismos valores, empleando métodos que no requieren ayuda alguna de la Geometría.

$$
\text { *** }
$$

En el estudio del doctor $J$. Alvarez Lleras publicado en la Revista de la Academia Colombiana de Ciencias Exactas, Físicas y Naturales [159], 474, en el cual analiza la Nota de Garavito que estamos comentando, se lee lo siguiente:

"El fin primordial del estudio de Garavito es demostrar que las fórmulas de la Trigonometría correspondiente a la Geometría de Lobatchewsky son las de la Trigonometría esférica imaginaria y que Lobatchewsky incurrió en un error de criterio o aparentó incurrir en él, al haber raciocinado geométricamente cuando el valor de sus deducciones era puramente analítico. Para llegar a tal resultado el doctor Garavito consideró las variaciones periódicas de cierta distancia $\approx$ comprendida entre un punto fuera de una recta y otro que se desaloja sobre ésta hasta el infinito. Del estudio de esas variaciones llegó a la conclusión de que Lobatchevsky dio de manos a boca con el Postulado de Euclides "porque no había razonado con rectas situadas en un plano, sino sobre otra clase de líneas y superficies". "¿Cuáles eran esas superficies y esas líneas?" continúa diciendo el doctor Garavito: En sus raciocinios Lobattcheffsky había encontrado que la suma de los tres ángulos de un triángulo era menor que dos rectos, precisamente lo contrario de lo que acontece con los triángulos esféricos en donde el exceso esférico es la relación del área del triángulo al cuadrado del radio de la esfera. Si pues el radio de la esfera se hiciese imaginario, su cuadrado se haría negativo y el exceso esférico se convertirá en de- fecto, tal y conforme corresponde al caso estudiado. Lobattcheffsky había pues razonado sobre una esfera imaginaria considerada como plano $y$ con ćrculos máximos de tal esfera considerados como rectas".

"Creemos - concluye Alvarez Lleras - que lo transcrito bastará para indicar dónde está el mérito del matemático colombiano cuando trató, antes que nadie de estas cuestiones".

$Y$ añade en nota:

"En un libro reciente de Picard se habla ya de estas cosas tocándolas incidentalmente; empero la propiedad del descubrimiento pertenece exclusivamente al doctor Garavito".

Estas conclusiones del doctor Alvarez Lleras son inadmisibles, pues, casi un siglo antes de que eseribiera Garavito, había hecho Taurinus la misma consideración de la esfera de radio imaginario en 1825 [127], 67; [133], 277; lo mismo que Lambert para el área del triángulo en la hipótesis del ángulo agudo [133], 267. También Lobatchewsky, en su célebre Memoria de 1840 [17], 34, deduce las formulas de la Trigonometría esférica de las que expresan en su sistema de geometría las relaciones entre los lados $a, b, c$ y los ángulos $A, B, C$ de un triángulo rectilíneo, sustituyendo en estas últimas $a, b, c$ por $a i, b i$, ci. Lo mismo hizo Bolyai en 1832 [22], 242; [39], 137.

Por otra parte, la generalización de esta idea o sea la célebre interpretación de Beltrami (18351900 ), fue publicada en 1868, es decir medio siglo antes de que viera la luz el estudio de Garavito que hemos analizado.

El estudio del ilustre geómetra italiano tiene por título Saggio di Interpretatione della Geometria non-euclidea y apareció en el t. VI del Giornale di Matematiche. Mostró Beltrami que todas las proposiciones de la Geometría plana de Lobatcherosky son válidas en el espacio euclidiano sobre las superficies de curvatura constante negativa [83], 33; [106], 13. Russell hace notar que es extraordinario que esta interpretación que Riemann conocía y quizás también Gauss, haya estado durante tantos años sin demostración explícita. Agrega que esto es tanto más raro cuanto que la Geometría imaginaria de Lobatchewsky aparecí en el tomo XVII del Journal de Crelle (1837) y Minding había mostrado en el tomo XIX del mismo perí́dico que la Geometría de las superficies de curvatura constante negativa en particular en lo que respecta a los triángulos geodésicos, puede deducirse de la de la esfera dando al radio un valor puramente imaginario $i a$.

Este resultado, como ya hemos visto, había sido obtenido por Lobatchewsky y sin embargo - agrega Russelll - se necesitaron 30 años (hasta 1868) para que el conocimiento de esta relación fuese general [V. [111], 74].

Sobre el mismo asunto dice el eminente matemá. tico francés Darboux en su monumental obra Théo. rie des Surfaces [58], 394, lo siguiente: 
"Esta teoría más general a la cual Lobatchewsky había dado el nombre de Pangeometría y que se designa hoy con el nombre de Geometría no euclidiana, concuerda enteramente, en el caso del plano, con la que acabamos de desarrollar para las superficies de curvatura constante negativa. Esta observación desarrollada de una manera completa por Beltrami, se justifica de la manera siguiente: Se reconoce que todas las propiedades del Tratado de Euclides que reposan sobre la noción del cambio de lugar de una figura invariable se aplican a las diversas superficies de curvatura constante, con tal de que se reemplacen las rectas del plano por las geodésicas de la superficie. El hecho, admitido en los elementos, de que no se puede llevar más de una recta por dos puntos excluye las superficies de curvatura positiva. Se sigue de alli que toda geometría en la cual no se añada el postulado de Euclides a los hechos anteriormente admitidos deberá convenir a las superficies de curvatura constante negativa lo mismo que al plano. Tal es, en rasgos generales, la explicación que debemos a Beltrami de la analogía completa que existe entre la Geometría enclidiana del plano y la de las superficies de curvatura constante negativa".

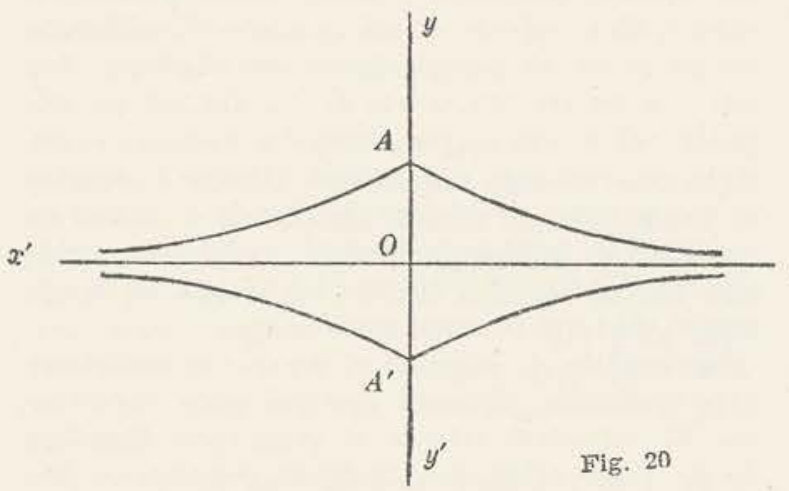

Una superfice de curvatura constante negativa es, por ejemplo, la pseudoesfera, superficie engendrada por la revolución de la tractriz o traztriz alrededor de su asíntota. La tractriz tiene la forma indicada en la figura 20 y tiene por ecuación

$$
x=a \log \frac{a+\sqrt{a^{2}-y^{2}}}{y}-\sqrt{a^{2}-y^{2}} .
$$

Esta curva es tal que los segmentos de tangente comprendidos entre el punto de contacto $\mathrm{y}$ el eje $\mathrm{O} x$ tiene una longitud constante $a$. El punto $A$ es de retroceso $\left({ }^{*}\right)$.

Como se ve por lo expuesto, es de toda imposibilidad atribuír a Garavito el descubrimiento de la interpretación de la Geometría hiperbólica por medio de las superficies de curvatura constante negativa, como lo hace Alvarez Lteras. Por otra parte, aunque Garavito no cita ninguna obra, dice textualmente:

(*) Ya hemos dicho que esta superficie no ofrece, en la Geometría euclidiana, la imagen completa del plano de Lobatchewsky. La métrica de esta superficie es la misma de la Geometría plana de Lobatchewsky, solamente en una cierta región comprendida entre dos aristas de retroceso.
"De esta fórmula pueden deducirse las tres fórmulas fundamentales de la Trigonometría esférica imaginaria como lo ha hecho Lobattcheffsky". De modo que, por confesión de Garavito mismo, él no hizo sino repetir lo que había ya hecho Lobatchewsky, es decir, sustituír el radio real $R$ por otro imaginario, o sea reemplazar $R$ por $R \sqrt{-1}$.

$$
\text { * * }
$$

Dice Garavito: "Gauss, Lobatchewsky y Riemann $\mathrm{y}$ en general los que han estudiado a fondo $\mathrm{y}$ detenidamente el asunto, han tenido forzosamente que llegar a las mismas conclusiones a que hemos llegado nosotros respecto del postulado de Euclides. Habiendo tropezado aquellos sabios con un interesante acertijo, se guardaron de aclararlo para dejar un motivo de entretenimiento a los curiosos, presentando el enigma bajo la forma de verosimilitud de otras geometrías planas no euclídeas".

Esta opinión no tiene nigún fundamento desde el punto de vista científico, pues vimos ya que las conclusiones de Garavito respecto del postulado de Euclides son falsas y mal podrían haberlas imaginado o aceptado sabios de la talla de los nombrados. Aun cuando hoy día no se discute ya el asunto de que se trata, basta para refutar a Garavito citar los escritos de Lobatchewsky y de Riemann y en lo que respecta a Gauss, leer su correspondencia con Schumacher y Wolfgang Bolyai.

En carta a Schumacher fechada en Gotinga el 28 de noviembre de 1846 dice Gauss:

"He tenido últimamente ocasión de releer el folleto de Lobatchewsky intitulado: Geometrische Untersuchungen zur Theorie der Parallellinien. Este opúsculo contiene los elementos de la Geometría que debería existir y cuyo desarrollo formaría un encadenamiento riguroso, si la Geometría euclidiana no fuera verdadera. Un tal Schweikardt (antes vivía en Marbourg, ahora es profesor de jurisprudencia en Koenisberg) ha dado a esta geometría el nombre de Geometría astral. Lobatchewsky el de Geometría imaginaria. Usted sabe que desde hace cincuenta y cuatro años (desde 1792) tengo las mismas convicciones, sin hablar aquí de ciertos desarrollos que han adquirido después mis ideas sobre esta materia. No he hallado, pues, en la obra de Lobatchensky ningún hecho nuevo para mí pero la exposición es enteramente diferente de la que había yo proyectado, $\mathrm{y}$ el autor ha tratado la materia magistralmente y con verdadero espíritu geométrico. Creo deber llamar su atención sobre este libro, cuya lectura no dejará de causarle el más vivo placer" [17], 41.

Leamos ahora la carta de Gauss a su amigo Wolfgang Bolyai, fechada el 6 de marzo de 1832, en la cual se refiere al célebre

Appendix, scientiam spatii absolute veram exhibens, a veritate aut falsitate axiomatis $X I$ Euclidei (a priori haud aunquam decidenda) independentem; adjecta, ad casum falsitatis, quadratura circuli geometrica.

de Juan Bolyai, hijo de Wolfgang: 
... "Hablemos ahora un poco del trabajo de tu hijo. Si comienzo diciendo que yo no pucdo alabar cse trabajo, quedarás por un instante asombrado; pero no puedo decir otra cosa; alabarlo sería alabarme; en efecto, el fondo completo de la Obra, el camino seguido por tu hijo, los resultados que él obtiene, coinciden casi enteramente con mis propias meditaciones que han ocupado en parte mi espiritu desde hace treinta o treinta y cinco años. Esto me ha dejado completamente estupefacto. Por lo que respecta a mi trabajo personal, sobre el cual conservo poca cosa por escrito, mi intención era de no dejar publicar nada mientras viva. En efecto, la mayor parte de los hombres no tiene un juicio seguro sobre los asuntos de que se trata $y$ he encontrado solamente muy pocos que mostrasen interés particular acerca de lo que les he comunicado a ese respecto. Para poder tener este interés es necesario haber antes sentido hondamente las imperfecciones esenciales y en estas materias casi todos los hombres están en una oscuridad completa. Tenía por. el contrario, la idea de redactar, andando el tiempo, todo esto a fin de que por lo menos no pereciera conmigo".

"Ha sido pues para mí una agradable sorpresa ver que puedo ahora dispensarme de ese trabajo y me llena de extremo júbilo que sea precisamente el hijo de mi viejo amigo quien me haya tomado la delantera de un modo tan notable". [65], 17; [160], 12.

Como Garavito dice: "los geómetras kantianos que, antes de Lobatchevsky conferían a los axiomas la categoría de verdades necesarias, admitieron después la existencia lógica de espacios no euclideos!", parece interesante hacer ver que las ideas de Gauss respecto del espacio eran totalmente opuestas a las de Kant. En la misma carta de Gauss a W. Bolyai de 6 de marzo de 1832 [65], 20, dice:

"En la imposibilidad en que estamos de distinguir a priori entre $\Sigma$ y S (sistema enclidiano y noeuclidiano), se halla precisamente demostrado del modo más claro que Kant no tuvo razón al afirmar que el espacio es únicamente la forma de nuestra intuición. He indicado una razón igualmente convincente en una Nota publicada en Göttingische Gelehrte Anzeigen en 1830, Part. 64, p .625".

Gauss se refiere aquí al análisis de su Memoria: Theoria residuorum biquadraticorum inserto en la publicación mencionada. Dice en la pág. 637 (Gauss, Werke, t. II, p. 177; 1876) :

"Esta distinción entre la derecha y la izquierda sería... en si completamente determinada, si no obstante pudiésemos comunicar nuestra intuición de esta distinción a otros únicamente por una prueba que reposara sobre los seres materiales en presencia efectiva de los cuales nos halláramos". Y en nota al pie de la página agrega:

"Las dos observaciones han sido ya hechas por Kant, pero no se concibe cómo ese filósofo perspicaz podía creer que la primera demostraba su opinión que el espacio es solamente una forma de nuestra intuición exterior, puesto que la segunda observación demuestra tan claramente lo contrario y que el espacio debe tener una significación real independientemente de nuestro modo de intuición". [65], 21.

\section{CONCLUSION}

Como hemos visto, todos los intentos de demos. tración del célebre postulado $5^{\circ}$ de Euclides que, por otra parte, es muy interesante e instructivo analizar, se fundan en reemplazar el postulado que se quiere demostrar por otro postulado. Algunos antores lo han hecho conscientemente como, por ejemplo, Wallis al reemplazar el postulado de Euclides por el postulado de la similitud o posibilidad de la construcción de figuras semejantes y Legendre al sustituírlo por el postulado o principio de la homogencidad. Otros autores como, por ejemplo, Garavito, han creído haber demostrado el célebre postulado, porque no se han dado cuenta de la sustitución de postulados que han operado implícitamente.

El error fundamental de los que atacan los sistemas no-euclidianos es el de creer que el postulado de Euclides está contenido en la noción de línea recta, o que es una consecuencia de los otros axio. mas. Otro error fundamental es el de pensar que los espacios euclidiano y no-euclidiano pueden coexistir. Si el espacio en que vivimos es euclidiano no puede ser al mismo tiempo no-euclidiano. Por esto demuestran ignorancia del asunto los que creyendo haber echado por tierra los sistemas no-euclidianos, exclaman satisfechos: "Vuelve a ser cierto que la suma de los tres ángulos de un triángulo rectilíneo es igual a dos rectos". Podría respondérseles que jamás, en el sistema euclidiano, ha dejado de ser cierto el teorema mencionado.

La cuestión de saber si el espacio es euclidiano o no-euclidiano, tiene en realidad poca importancia. El verdadero interés, el gran valor filosófico de las Geometrías no-enclidianas consiste - dice Russell - en la posibilidad lógica de su existencia, aun en el caso improbable en que se pudiese demostrar rigurosamente que nuestro espacio es euclidiano [83], 125 .

Otro punto que atacan los enemigos de las geometrías no-euclidianas es el de la existencia de la constante espacial que algunos llaman "Ia misteriosa constante $k$ ". [126], 14. En las geometrías noeuclidianas la distancia $D$ entre dos puntos no está dada directamente por el valor de $D$ en función de las coordenadas, sino en la Geometría de Riomann por el valor de $\cos \frac{D}{k}$ y en la de Lobatchewsky por el de $\cosh \frac{D}{k}$ en función de las coordenadas de los dos puntos. Esta constante $k$, como dice Russell [33], 262 está implícitamente contenida en toda relación analítica no-euclidiana que contiene distancias, así como la constante cuatro ángulos rectos está incluída en toda ecuación de geometría euclidiana o no-euclidiana que contiene ángulos. La cantidad $\frac{1}{k^{2}}$ en la Geometría de Riemann y $-\frac{1}{k^{2}}$ en la de Lobatchersky es lo que se llama la curvatura 
total, derivada de la fórmula del arco infinitesimal por la misma fórmula que ha dado Gauss para la curvatura total de una superficie $\left(^{\left(\frac{n}{*}\right.}\right)$.

Los filósofos que no son matemáticos, como Lotze, han sido en general, enemigos acérrimos de la metageometría. Lotze expresaba la esperanza de que la Filosofía no se dejara dominar en esta materia por las Matemáticas. Russell, que es filósofo y matemático, dijo: "Es necesario, por el contrario, alegrarse de que las Matemáticas no se hayan dejado dominar por la Filosofía y que hayan desarrollado libremente un sistema importante y consecuente consigo mismo, que merece por su sutil análisis de los elementos lógicos y de hecho, la gratitud de todos los que buscan una filosofía del espacio". [83], 139.

\section{NOTAS SOBRE EUCLIDES Y LAS EDICIONES DE LOS ELEMENTOS}

Se ha confundido frecuentemente al geómetra Euclides con Euclides de Megara fundador de una secta. Dice Montucla, "célebre más bien que por sus progresos en la investigación de la verdad, por su invención de sofismas y su pasión por la disputa".

Euclides de Megara fue uno de los primeros anditores de Sócrates; mientras que el geómetra era contemporáneo del primer Ptolomeo y por consiguiente vivió un siglo más tarde que aquél (cerca de 300 años a. J. C.).

No se sabe con seguridad cuál fue la patria del geómetra Euclides. Parece que vivió primeramente en Grecia y estudió en Atenas con discípulos de Platón. Después se fijó en Alejandría llamado por Ptolomeo. Este le preguntó si no había camino más fácil, menos espinoso que el ordinario para estudiar la Geometría. "No, príncipe - respondió Euclides - no existe ninguno hecho expresamente para los reyes". [7], 204.

Los célebres Elementos se componen de trece libros a los cuales se añaden ordinariamente otros dos que se atribuyen a Hypsicles, geómetra de Alejandría que vivió ciento cincuenta años después de Euclides.

Theon de Alejandría (320-395) fue el primer comentador de Euclides y luego Proclus (412-485) y Eneas de Hierápolis.

El árabe Thebith ben Corrah tradujo y revisó los Elementos en el siglo IX.

El célebre astrónomo y geómetra persa Nassir. Eddin (1225-1274) fue el principal comentador de Euclides y su sabio comentario en árabe fue publicado en 1594 en la magnífica imprenta de los Médicis.

Los hebreos Moses Aban-Tibon e Isaac ben-Honain hicieron traducciones de Euclides que se conservan en manuscritos en algunas bibliotecas.

Adelard (AEthelhard) de Bath en Inglaterra y Campanus de Novara (Italia) tradujeron los Ele. mentos en los siglos XII y XIII de las versiones

(*) V. para la fórmula de la curvatura total, por ejemplo, DUARTE, Análisỉs Infinitesimal, No 142, p. 281. Caracas, 1943. árabes. Por estas traducciones los latinos comenzaron a conocer a Euclides; hasta entonces los únicos autores conocidos en Geometría eran Boëce y los Principiis geometriae de San Agustín. La obra de Adelard no se conoce sino en manuscrito. La de Campano (siglo XIII) fue la base para la mayor parte de las traducciones latinas de fines del siglo XV y principios del XVI.

Casi medio siglo después del descubrimiento de la imprenta aparecio en 1482 la editio princeps de Euclides, in folio, publicada en Venecia, de acuerdo con la traducción de Campano por Radtolt de Ausburgo, impresor célebre.

En 1489 se publicó otra edición, con el comentario de Campano, hecha en Vicencio por los impresores asociados Leonardo de Basilea y Guillaume de Pavia.

Zamberti de Venecia publicó otra edición latina de los Elementos y de otros escritos de Euclides con el título Euclides Opera, Bartholomaeo Zamberto interprete, Venetia in folio, 1505.

Esta traducción fue de nuevo impresa en Basilea en 1537 (in folio) por el impresor Hervage y nuevamente en 1565 .

En 1509 apareció en Venecia la bella edición in folio de Lucas Pacioli, de Borgo San Sepolcro, quien utilizó la versión de Campano: Euclides megarensis mathematicorumque omnium sine controuersia principis opus. Campano interprete fidissimo translata... Lucks Paciolus theologus insignis: altissima mathematicae disciplinarum scientia rarissimus... Venetiis, Paganinus 1509, in fol. (Título en rojo y negro, caracteres góticos y grabados en madera).

En 1516, Jacobo Faber de Etaples, publicó en París por el editor Henri Etienne una edición latina de los Elementos, traducida del griego. Ella contiene además del comentario de Theon, las notas de Campano y de Zamberit y no es una simple reimpresión de la edición dada por este último.

En 1533 apareció en Basilea la primera edición griega de los Elementos hecha por Simón Gryncueus en la célebre imprenta de $J$. Hervaye. Esta edición presenta el texto griego de Euclides según Theon y contiene los cuatro libros del Comentario de Proclus sobre el Primer Libro.

Commandin dio en 1572, en Pesaro, una traducción latina de los Elementos (in folio) y en 1575 una edición italiana hecha en Urbino, reimpresa en Pesaro en 1619 con adiciones y correcciones.

El célebre matemático Tartalea (Tartaglia) publicó en Venecia en 1543 una edición italiana de Euclides: Euclides. Solo introduttore delle Scientie Mathematice diligentemente rassetato et alla integrita ridotto per Nicolo Tartalea Bresciano. Secondo le due tradottioni et di latino in volgar tradotto, con una ampla espositione di novo aggionta Vinegia, Vent. Ruffinelli, 1543. Fue reimpresa en Chiara con notas de Talmente en 1565.

En 1566 apareció la edición latina (in folio) de De Foix-Candalle con un décimo libro sobre los sólidos regulares; fue reimpresa en 1578 y aumentada de dos nuevos libros sobre los mismos sólidos. 
El P. Clavius, s. J., dio una edición de Euclides en 2 vols. in $8^{\circ}$, en Roma, 1574, con comentarios. Esta edición tuvo numerosas reimpresiones.

En 1576 apareció la edición española: Los Seis Libros Primeros de la geometría de Euclides. Traduzidos en Lêngua española por Rodrigo Camorano, Astrologo y Mathematico. Sevilla.

Después se publicó la edición francesa: Les Quinze livres des Elémens d'Euclide. Traduicts du latin en François par D. Henrion, Mathematicien, París, 1615.

Barrow publicó una edición latina en 1659 y Keil otra, en Oxford, en 1701 (in $8^{\circ}$ ).

La magnífica edición en griego y latín de David Gregory fue publicada en Oxford en 1703 (in folio) con el título Euclides quae supersunt omnia (*).

En 1756 Robert Simpson publicó en Glasgow una edición latina (in $4^{\circ}$ ) a la cual siguió una edición inglesa.

Después vienen las ediciones modernas:

Les CEuvres d'Euclide, traduites en Latin et en F'rançais, d'après un manuscrit grec très ancien qui était inconnu jusqu’à nos jours. Par F. Peyrard, Traducteur des œuvres d'Archimède. París, 1814, 1816, 1818. (Edición en griego, latín y fracés, 3 vols. in $\left.8^{\circ}\right)$.

(*) En esta edición los postulados 4, 5, 6 están marcados $10,11,12$. Por esto el postulado de Euclides se designa a veces por "axioma XI", como en el título del Appendix de Bolyai.
Les Guvres d'Euclide, traduites littéralement d'après un manuscrit grec très ancien resté inconnu jusqu'a nos jours. Par E. Peyrard, París, 1819. (Edición solamente en francés, en 1 vol. in $4^{\circ}$ ).

Estas traducciones de Peyrard están consideradas como las más completas y las mejores que existen: "edición muy preciosa, considerada como la mejor y la más completa que se posee". (Biogr. Gen.).

El manuscrito antiguo utilizado en esta traduc. ción es el No 190 de la Biblioteca del Vaticano, el cual fue enviado a París por Monge y Berthollet, cuando la ocupación de Roma, en 1796. Cuando Francia debió devolver los tesoros incautados, el Papa (Pío VII) permitió, a pedido del Gobierno francés, que Peyrard conservase el manuscrito hasta terminar la traducción.

Euclidis Elementa ex optimis libris in usum Tironum Graece edita ab Ernesto Ferdinando August Berlín, 1826-1829.

Euclidis Elementa, edidit et latine interpretatus est J. L. Heiberg. Lipsiae 1883-1888. 5 vols. in $8^{\circ}$ [Vol. I, Libri 1-4; Vol. II, Libri 5-9; Vol. III, Libri 10; Vol. IV, Libri 11-13; Vol. V, Libri 14-15]; con un total de CLVII + 2348 págs.

The Thirteen Books of Euclid's Elements. Translated from the text of Heiberg with introduction and commentary, by T. L. Heath. 3. vols. in 8, Cambridge, 1908.

\section{BIBLIOGRAFIA}

[1] Clavio, C. - Operetta della linee rette equidistanti et non equidistanti, Bolonia, 1603.

[2] Borelli, J. A. - Euclides restitutus. Pisa, 1658.

[3] Giordano, V. - Euclide restituto overo gli antichi elementi geometrici ristaurati e facilitati. Roma, 1680.

[4] Wallis, J. - Opera Mathematica, t. II, p. 669-678. (De postulato Quinto) Oxoniæ, 1693.

[5] Klügel, G. S. - Conatuum præcipuorum theoriam parallelarum demostrandi recensio, quam publico examini submittent A. G. Kaestner et auctor respondens G. S. Klügel. Göttingen, 1763.

[6] D'Alembert, J. - Encyclopédie Méthodique Mathématique, t. II, p. 519, art. Parallèles. París, 1785.

[7] Montucla, J. F. - Histoire des Mathématiques. 4 vols. in 4 \%, t. I. Paris an VII, (1799), p. 204-214.

[8] Hoffmann, J. - Kritik der Parallelentheorie. Jena, 1807.

[9] Flauti, V. - Nuova dimostrazione del postulato quinto. Nápoles, 1818.

[10] Peyrard, F. - Les, CEuvres d'Euclide. París, 1819, p. 2.

[11] Legendre, A. M. - Eléments de Géométrie, $12^{\circ}$ ed. Paris, 1823 , p. $20 ; 273-282$.

[12] Legendre, A. M. - Reflexions sur différentes maniéres de démontrer la théorie des parallèles ou le théorème sur la somme des trois angles du triangle. Mém. de l'Acad. des Sciences. París, t. XIII, 1833.

[13] Allgemeine Encyklopaedie der Wissenschaften und Kuenste $3^{a}$ Sección. Leipzig, 1835. Artículo de Sohnke en el que citan 92 obras sobre la teoría de las paralelas.

[14] Bouniakofsky, M. - Sobre la teoría de las paralelas (en ruso) 1853. Artículo de 15 págs. en el que se hallan las críticas de las demostraciones del postulado de Euclides de Proclus, Nassir-Eddin, Castillon, Clavius, Simpson, Bertrand (de Ginebra), Gouriew, Schulten, Cristian, Tatarinow y Legendre. (Según A. Vassilief. L'Intermediare des Mathématiciens. t. II, p. 405).

[15] De Tilly, J. M. - Recherches sur les élements de la Géométrie. Bruxelles, 1860.
[16] Hoüel, J. - Essai d'une exposition rationnelle des principes fondamentaux de la Géométrie élémentaire. Arch. d. Math. u. Phys. t. 40. 1863.

[17] Lobatehewsky, N. I. - Etudes géométriques sur la théorie des parallèles, trad. Hoüel. París, 1866, p. 34.

[18] Lobatchewsky, N. I. - Pangeometria, trad. italiana de la edición francesa de Kasan de 1855 (Giorn. di Math. t. V). Nápoles, 1867. p. 1-64.

[19] Forti, A. - Intorno a la Geometria imaginaria o non euclidiana. Considerazioni storico-critiche. Rivista Bolognese di scienze, lettere, etc. t. II. p. 171. 1867.

[20] Carton, J. - Vrais principes de la Géométrie Euclidienne et preuves de l'impossibilité de la Géométrie non Euclidienne. París, in $8^{\circ}$ (s. d.) (1867?).

[21] Battaglini, G. - Sulla Geometria immaginaria di Lo batchewsky. Gior. di Mat. t. V. p. 217-231. 1867.

[22] Bolyai, J. - La Science Absolute de l'Espace indépendante de la vérité ou de la fausseté de l'Axio. me IX d'Euclide (que l'on ne pourra jamais établir a priori). Trad. Hoüel, París, 1868 (reimpreso por Hermann en 1896). p, 219; 242-248.

[23] Forti, A. - Intorno alla vita ed agli seritii di Wolfgang e Giovanni Bolyai di Bolya, matematici ungheresi. Bolletino di Bibliografia e di Storia delle scienze Mat. e Fisiche. t. I, p. 277. 1869.

[24] Hoüel, J. - Sur l'impossibilité de démontrer par une construction plane le postulatum d'Euclide. Nouvelles Annales de Math. $2^{\mathrm{a}}$ série. t. IX, p. 93 . Pa rís, 1870.

[25] Hoüel, J. - Notices sur la vie et les travaux de N. J. Lobatschefskij. Bulletin des Sc. Math. t. I; p. $66 ; 324 ; 384$. París, 1870.

[26] Klein, F. - Ueber die sogenannte nichteuklidische Geometrie. Mathematísche Annalen. t. IV, 1871; t. VI, 1873 ; t. VII, 1874.

[27] Flye Saint-Marie, C. - Etudes analytiques sur la théorie des parallèles. París, 1871.

[28] Frischauf, J. - Absolute Geometrie nach J. Bolyai. Leipsig, 1872 . 
[29] Friedleins, G. - Procli Diadochi in primum Euclidis elementorum librum Commentarii. Leipzig, 1873.

[30] Clifford, W. K. - Preliminary Sketch of Biquaternions. Proc. London Math. Society, 1873.

[31] Frischauf, J. - Elemente der Absolute Geometrie. Leipzig, 1876.

[32] Lagrange, J. L. - Cuuvres, t. VIII, p. 331-363. París, 1877.

[33] De Tilly, J. M. - Essai sur les principes fondamentaux de la Géométrie et de la Mécanique. Mémoires de la Soc. des Sc. de Bordeaux. t. III, 1878.

[34] Halsted, G. B. - American Journal of Math. t. I, II, (Bibliografía de literatura no enclidiana). 18781879.

[35] Kettner, W. - Beschouwingen over de theorie der evenwipdige lijnen als Grondstag der Meetkunde (Consideraciones solbre la teoría de las paralelas como fundamento de la Geometría) Leyde, 1879. Se discuten en este trabajo varias demostraciones del postulado de Euclides. L'Intermédiare des Math. t. II, p. 405 .

[36] Hoüel, J. - Essai critique sur les principes fondamentaux de la Géométrie élémentaire. $2^{e}$ éd. Paris, 1883.

[37] Tannery, P. - Sur l'authenticité des axiomes d'Euclíde Bull. des Sc. Math. $2^{e}$ série. t. VIII, p. 162 Paris, 1884.

[38] Killing, W. - Die nichteuklidischen Ranmformen in analytischer Behandlung, Leipzig, 1885.

[39] Riccardi, P. - Saggio di una bibliografía Euclidea. Mem. de I'Ist. di Bologna, serie 5, t. I. p. 27-34, 1887 .

[40] Laurent, H. - Traité d'Analyse. 7 vols. in 8 t. III, p. 381. París, 1888.

[41] Cirodde, P. L. - Lecciones de Geometría. Trad. Barbery. $14^{a}$ ed. Madrid, 1888. p. 18.

[42] Liard, L. - Des définitions géométriques et des définitions empiriques. Paris, $2^{\mathrm{a}}$ ed. 1888

[43] Cayley, A. - Collected Math. Papers, t. II. Cambridge, 1889. t. XII, 1897. p. 220-238.

[44] Calinon, A. - Les espaces géométriques. Revue Philosophique, París, 1889.

[45] De Broglie, Abbé. - La Géométrie non-euclidienne. Annales de Phil. Chrétienne. París, 1890.

[46] Andrade, J. - Les bases expérimentales de la Géométrie euclidienne. Rev. Phil. París, 1890-1891.

[47] Fontené, G. - L'Hyperespace à $\mathrm{n}-1$ dimensions. $\mathrm{Pa}$ rís, 1892.

[48] Gérard, L. - Sur la Géométrie non-euclidienne, París, 1892.

[49] Couturat, L - Note sur la Géométrie non-euclidienne et la relativité de l'espace. Revue de Métaphysique et de Morale. París, 1893.

[50] Karagiannides, A. - Die Nichteuklidische Geometrie. Berlín, 1893.

[51] De Tilly, J. M. - Essai de Géométrie analytique générale. Mém. de l'Acad. Royale de Belgique. t. XLVIII; Mathesis, 1893.

[52] Lechalas, G. - Note sur la Géométrie non-euclidienne et le principe de similitude. Rev. de Métaphysique et de Morale. París, 1893.

[53] Lie, S. und Engel, F. - Theorie der Transformations gruppen, 3 vols. in 8 ․ t. III. Leipzig. 1893. p. 394 ; p. 535.

[54]Delboeuf, J. - L'ancienne et les nouvelles géométries. Rev. Phil. 1893-1895.

[55] Killing, W. - Einführung in die Grundlagen der Geometrie. 2 vols. in 8. Leipzig, 1893-1898.

[56] Burnside, W. - On the Kinematics of non-Euclidean Space. Proc. London Math. Soc. vol. XXVI, 1894.

[57] Veronese, G. - Grundzuege der Geometrie. Trad. A. Schepp. Leipzig, 1894

[58] Darboux, G. - Leçons sur la théorie générale des surfaces. 4 vols. in 8. t. III, p. 394-419. Paris, 1894 .

[59] Stäckel und Engel. - Theorie der Parallellinien von Fuklid bis auf Gauss. Leipzig, 1895 .

[60] Lechalas, G. - La courbure et la distance en Géomé trie générale. Rev. de Métaphysique et de Morale. París, 1896.

[61] Dauge, F. - Cours de Méthodologie mathématique París, 1896. Se indican varias demostraciones de 5 postulado.

[62] Loria, G. - Il passato ed il presente delle principal teorie geometriche. $2^{\mathrm{a}}$ ed. p. 281-302. Turín, 1896.
[63] Couturat, L. - De l'infini mathématique, p. 233, París, 1896.

[64] Bonnel, J. F. - Les hypothèses dans la Géométrie. Paris, 1897.

[65] Stäekel und Engel. - Gauss, les deux Bolyai et la Géométrie non-euclidienne. Trad. Laugel. París, 1897. p. 21.

[66] Stäekel und Engel. - Nicolaj Iwanowitsch Lobatschefsky. Leipzig, 1898.

[67] Klein, F. - Conférences de Chicago. Trad. Laugel. París, 1898. p. 85; 121 .

[68] Poincaré, H. - Des fondements de la Géométrie. Trad. francesa por L. Rougier de una Memoria de Poincaré publicada en la revista americana "The Monist" en enero de 1898. París (s. d.) p. 5-64.

[69] Mansion, P. - Mélanges Mathématiques. Gand, 1898. Volumen que contiene artículos de 1882 a 1898. p. $33-69 ; 89-92 ; 122-144 ; 17-34$ ( $2^{\mathrm{a}}$ parte $) ; 43-54$ V. también Revue des Questions Scientifiques, Bruxelles, $t$. XXXVII donde da Mansion una lista bibilográfica sobre las geometrías no enclidianas.

[70] Riemann, B. - CEurres Mathématiques. Trad. Laugel. París, 1898. p. 280-299.

[71] Stäckel, P. - Franz Adolph Taurinus. Abhandlungen zur Geschichte der Math. t. IX. p. 397-427. Leipzig. 1899.

[72] Darboux, G. - Sur une classe remarquable de courbes et de surfaces algébriques. $2^{\mathrm{e}}$ ed. París, 1899. p. $208-217 ; 232-235$.

[73] Dehn, M. - Die Legendreschen Sätze neber die Winkelsumme in Dreleck. Math. Annalen. t. 33, p. $405-439.1900$.

[74] Gauss, C. F. - Werke, t VIII. p. 167; 234; 255; 267. Gottingen, 1900.

[75] Klein, F. - Zur Nichteuklidschen Geometrie. Math. Ann. t. 37, p. 544-572. 1900.

[76] Andrade, J. - L'Enseignement de la Géométrie et les géométries non-enclidiennes. L'Enseignement Math. IIe Année. p. 114-126. 1900.

[77] Frolov, M. - Considérations sur la Géométrie noneuclidienne. L'Eseignement Math. IIe Année. p. 179187. 1900.

[78] Frolov, M. - Nouvelles considérations sur les géométries non-euclidiennes. L'Enseignement Math. IIe Année. p. 293-298, 1900.

[79] Andrade, J. - Euclidien et Non-Euclidien. L'Enseignement. IIe Année. p. 298-300. 1900.

[80] Barbarin, P. - A propos d'un article de M. Frolov. L'Ens. Math. IIe Année p. 306. 1900.

[81] Tikhomandritzki, M. - Sur le postulatum d'Euclide L'Ens. Math. IIe Année. p. 385-388, 1900.

[82] Mansion, P. - Lettre au Directeur de l'Enseignement Mathématique. IIe Année. p. 457. 1900.

[83] Russell, Bertrand A. W. - Essai sur les fondements de la Géométrie. Trad. Cadenat. París, 1901. p. $33-35 ; 139 ; 189 ; 214-215 ; 262$.

[84] Isely, L. - Histoire des Mathématiques dans la Suisse Française. Neuchatel, 1901, p. 87.

[85] Wickersheimer, E. - Sur le postulatum des parallèles. L'Ens. Math. IIIe Année, p. 279-285. 1901.

[86] Bonola, R. - Index Operum ad geometrian absolutum spectantium. Leipzig, 1902-1903.

[87] Pietzker, F. - Considérations sur la nature de l'espace. L'Ens. Math. IVe Année. p. 76-110. 1902.

[89] Laurent, H. - A propos d'un article de M. Pietzker L'Ens. Math. IVe Année. p. 434-437. 1902.

[90'] Barbarin, P. - Sur un quadrilatère birectangle. L'Ens. Math. IVe Année. p. 438-444. 1902.

[91] Hilbert, D. - Neue Begruendung der Bolyai.-Lobatschefskijschen Geometrie. Math. Ann. t. 57, 1903.

[92] Segre, C. - Congetture intorno alla influenza di Gerolamo Saccheri sulla formazione della Geometria non euclidea. Atti Acc. Sc. di Torino, t. 38, 1903.

[93] Combebiae, G. - L'Espace est-il enclidien? L'Ens. Math. Ve Année. p. 157-177; 262-278. 1903.

[94] Pesloüan, L. de. - Sur la nécessité du Postulat d'Euclide. L'Ens. Math. Ve Année. p. 288-293. 1903.

[95] Bonola, R. - A propos d'un récent exposé des principes de la Géométrie non-euclidienne. L'Ens. Math. Ve Année. p. 317-325. 1903.

[96] Commolet, J. B. - Théorie des paralléles euclidiennes. L'Éns. Math. Ve Année. p. 326-331. 1903. 
[97] Lechalas, G. - Introduction à la Géométrie générale. París, 1904. p. 40-42; 50-52.

[98] Bordage, E. - Sur un théoréme de la Géométrie rimannienne. L'Ens. Math. VIe Année, p. 239-241. 1904.

[99] Bonola, R. - I teoremi del Padre Gerolamo Saccheri sulla somma degli angoli di un triangolo e la ricerche di M. Dehn. Rend. Ist. Lombardo, serie 11, vol. 38. 1905.

[100] Bonola, R. - La trigonometria assoluta secondo Giovanni Bolyai Rend. Ist. Lombardo, vol. 38. 1905.

[101] Vahlen, K. Th. - Abstrakte Geometrie. Untersuchungen ueber die Grundlagen der Euklidischen und nichteuklidischen Geometrie. Leipzig, 1905.

[102] Bonola, R. - Un teorema de Giordano Vitale de Bitonto sulle rette equidistanti. Bolletino di Bibliogr. e Storia delle Sc. Math. 1905.

[103] Study, E. - Ueber Nicht-Euklidische und Liniengeometrie. Jahr. der Deutsche Math. Vereinigung. XV. 1906.

[104] Bonola, R. - La Geometria non-euclidea. Bologna. 1906 .

[105] Laurent, H. - La Géométrie analytique générale. Paris, 1906. p. 5; 29; 137 .

[106] Barbarin, P. - La Géométrie non-euclidienne. $2^{e}$ ed. París, 1907 , p. $9 ; 49-54$.

[107] Kantor, M. - Vorlesungen ueber Geschichte der Mathematik. 4 vols. in 8. t. I. Leipzig, 1907.

[108] Haisted, G. B. - La Sphérique non-euclidienne. L'Ens. Math. Xe Année. 1908, p. 97-111.

[109] Coolidge, J. L. - The elements of no-euclidean geometry. Oxford, 1909.

[110] Schur, F. - Grundlagen der Geometrie. Leipzig, 1909.

[111] Picard, E. - La Science moderne et son état actuel. París, 1909. p. 67-81.

[112] Laurent, H. - Sur les principes fondamentaux de la Theorie des nombres et de la Géométríe. $2^{e}$ ed. Paris, 1911. p. $39 ; 43 ; 57$.

[113] Halsted, G. B. - Géométrie rationnelle. Trad. Barbarin. París, 1911. p. $220-260$

[114] Eneyelopédie des Sciences Mathématiques pures et appliquées publiée sous les auspices des Académies des Sciences de Göttingue, de Leipzig, de Münich et de Vienne. Ed. Française. t. III, vol. I, p. 1-64. Leipzig, 1911.

[115] Cailler, C. - Sur la notion de courbure et sur quelques points de Géométrie infinitésimale Non-Euclidienne. Mém. de la Soc. de Physique et d'Histoire Naturelle de Genéve. Vol. 37, fasc. 2. 1911.

[116] Rouché et Comberousse. - Traité de Géométrie. 8e ed. t. II. París, 1912. p. 575-593.

[117] Poincaré, H. — La Science et l'Hypothèse. París, 1912. p. $47-67$.

[118] Brunschvicg, L. - Les étapes de la Philosophie mathématique. París, 1912. p. 497-524.

[119] Hadamard, J. - Leçons de Géométrie élémentaire. 5 e ed. t. I. París, 1913, p. 286.

[120] Stäekel, P. - Urkunden zur Geschichte der Nichtenklidische und Engel, F. Geometrie, 2 vols. in 8. Leipzig, 1913.

[121] Ture, A. - Introduction élémentaire à la Géométrie Lobatschewskienne. Genéve, 1914. p. 62.

[122] Poincaré, H. - Sur les groupes kleinéens. Guvres, t. II. París, 1916. p. 23-25 [Comptes Rend. de l'Acad. des Sc. de París. t. 93, 1881].

[123] Rey Pastor, J. - Introducción a la Matemática superior. Madrid, 1916. p. 35-43.

[124] Rey Pastor, J. Fundamentos de la Geometría proyectiva superior. Madrid, 1916.

[125] Darboux, G. - Principes de Géométrie analytique. París, 1917. p. 2; 289-363.

[126] Garavito A., J. - Nota sobre las Geometrías planas no euclideas. Anales de Ingeniería. Bogotá, 1918. Separata, 1918. [Reproducida en Rev. de la Acad. Colomb. de Ciencias Exactas, Físicas y Nat. Vol. II, No 8. p. 566-572. Bogotá, 1939].

[127] Bonola, R. - Die Nichteuklidische Geometrie. Trad. Liebmann. 2e Aufl. Leipzig, 1919. p. 61; 107. [3 Aufl. 1921].
[128] Montessus de Ballore, $\mathbf{R}$ de. $-i$ Se puede vulgarizar la Matemática superior? Trad. Rev. Mat. H. A. t. I. Madrid, 1919. p. 81-88. [L'Ens. Math. IIIe Année 1901. p. 106-114].

[129] Boutroux, P. - Les principes de l'Analyse Mathématique. t. II. Paris, 1919, p. 208-214.

[130] Vessiot, E. - Leçons de Géométrie supérieure. París, 1919. p. $78-80$.

[131] Enriques, F. - Les concepts fondamentaux de la Science. Trad. Rougier. París, 1919. p. 5-83.

[132] Rougier, L. - La Philosophie géométrique d'Henry Poincaré. París, 1920.

[133] Enriques, F. - Cuestiones relativas a la Matemática elemental. Trad. de la Soc. Mat. Española, t. I. Valladolid, 1921. p. 260-379.

[134] Mae Leod, A. - Introduction à la Géométrie NonEuclidenne. París, 1922. p. 285; 309; 311; 253-284.

[135] Bianchi, L. - Lezioni di Geometria Diferenziale. 3 vols. in 8 . t. I. $3^{\mathrm{a}}$ ed. Bologna, 1922. p. $638 ; 642$.

[136] Picard, E. - Deux lecons sur certaines équations fonctionnelles et la Géométrie non-euclidienne. Bull. des Sc. Math. t. XLVI. Paris, 1922. p. 404-416; 425-432.

[137] Hilbert, D. - Grundlagen der Geometrie. 5 Aufl. Leipzig, 1922. p. 144-162.

[138] Cailler, Ch. - Introduction géométrique à la Mécanique rationnelle. Genève, 1924. p. $49 ; 202 ; 378$.

[139] Enriques, F. - Gli Elementi d'Euclide e la critica antica e moderna. 4 vols. in 8 . Roma e Bologna, 19251932.

[140] Barbarin, P. - La correspondance entre Hoüel et De Tilly. Bull. des Se. Math. t. 50. París, 1926. p. $50-64 ; 74-88$

[141] Einstein, A. - Geometría no enclidea y Física. Rev. Mat. H. A. $2^{\text {a }}$ serie. t. I. Madrid, 1926. p. 72-76.

[142] Gonseth, F. - Les fondements des Mathématiques. París, 1926. p. 75-115.

[143] Appell, P. - Traité de Mécanique Rationnelle. 5 vols. in 8. t. v. Paris, 1926. p. 173.

[144] Klein, F. - Vorlesungen neber Höhere Geometrie. 3 Aufl. Berlín, 1926.

[145] Jiménez Soto, F. - Nota sobre el postulado de Euclides. Rev. Mat. H. A. t. II. $2^{\mathrm{a}}$ serie. Madrid, 1927. p. 212-216.

[146] Barbarin, P. - La Géométrie Non-Euclidienne. 3e éd. París, 1928. p. 8; 52-58; 86-99; 113-168.

[147] Picard, E. - Lecons sur quelques équations fonctionnelles. París, 1928. p. 1-49.

[148] Barbarin, P. - Sur les images euclidennes du plan non-euelidien. Bull. des Sc. Math. t. 52. París, 1928 p. $317-319$.

[149] Barbarin, P. - Images euclidiennes des plans noneuclidiens. Atti del Congresso Internazionale dei Matematici. t. IV. Bologna, 1928, p. 61-67.

[150] Parfentieff, N. N. - La Philosophie de la Nature chez N. J. Lobatcewski. Atti del Congresso de Bologna. t. VI, 1928. p. $483-488$.

[151] Klein, F. - Vorlesungen ueber Nicht-Euklidische Geometrie. 2 vols. in 8. Berlín, 1928.

[152] Cartan, E. - Leçons sur la Géométrie des espaces de Riemann. París, 1928. p. 133-177.

[153] Bentelli, W. - Somengeometrie und Niche-Euklidische Geometrie. Comm. Math. Helvetici. Vol. I. Zürich, 1928. p. $42-63$.

[154] Costz, A. - As idéas fundamentaes da Mathematica. Río de Janeiro, 1929, p. 201-211.

[155] Schilling, F. - Projektive und Nichtenklidische Geometrie. 2 vols. in 8. Leipzig, 1931.

[156] Buhl, A. - Paul Barbarin. Bull. des Sc. Math. t. 56 . París, 1932, p. 72-78.

[157] Juvet, G. - La structure des nouvelles théories physiques. París, 1933. p. 156-163.

[158] Sehilling, F. - Die Pseudosphaere und die Nichtenklidische Geometrie. Leipzig, 1935.

[159] Alvarez Lleras, J. - Julio Garavito Armero. Ensayo biográfico y literario. Rev. Acad. Colomb. de Cien cias exactas, físicas y naturales. Vol. II. No 7. Bogotá, 1938. p. 474. (Critica a las Geometrías noeuclidianas). 
[160] Duarte, F. J. - C. F. Gauss. Rev. del Colegio de Ingenieros de Venezuela. Año XVI, No 129 . Caracas, 1938. p. 309. Separata, 1938.

[161] Bell, E. T. - Les grands mathématiciens. Trad. A. Gandillon. París, 1939. p. 320-333.

[162] Kerekjarto, B. de. - Nouvelle méthode d'édifier la géométrie plane de Bolyai et de Lobatchefsky. Comm. Math. Helvetici. Vol. 13. Zürich, 1940. p. $10-48$.

[163] Hempel, C. G. - Geometry and Empirical Science. The American Math. Monthly. Vol. 52, No 1, pág. 7-17. Chicago, 1945.

NOTA DE LA DIRECCION. - Hemos reproducido íntegramente este folleto del doctor Francis. co J. Duarte, publicado en Caracas en 1945, porque fuera de su gran mérito intrínseco contiene él algunas apreciaciones contrarias a Garavito, $y$ en estas páginas deben tener cabida opiniones de toda clase, aunque nos sean adversas. Tal reproducción es enteramente espontánea: nadie nos ha sugerido el hacerla, ni el propio autor del folleto, quien accedió a nuestros deseos por sentimientos de sim. patía hacia la Academia Colombiana de Ciencias Exactas, Físicas y Naturales, a la cual pertenece con el título de miembro correspondiente.

Al suministrar a nuestros lectores la copia atrás presentada, y que hemos verificado con nimio cuidado, nos permitimos recomendar su lectura por considerar que el estudio: "Notas históricas y bibliográficas sobre las Geometrías no euclidianas" es digno de la más grande atención. Su autor se muestra en este escrito como erudito de primera clase. Tal vez no se haya hecho nunca un trabajo limitado y abreviado sobre la materia con tal cantidad de información, que supone un espiritu benedictino de investigación y conocimientos matemáticos muy poco comunes. Merece por ello nuestro colega las más sinceras felicitaciones.

Pero si hemos puesto en la reproducción a que aludimos, el mayor cariño, obedeciendo a un sincero deseo de que sea este estudio ampliamente conocido y de que nuestros lectores sepan cuál es la crítica que el doctor Duarte endereza contra las opiniones de Garavito respecto de las Geometrías no-euclidianas, esto no obsta para que nos reservemos para algún otro lugar en donde podamos, con mayor espacio, refutar tal crítica.

Por lo pronto, muy respetuosamente observamos al doctor Duarte que nunca hemos entendido que Garavito se hubiera propuesto demostrar el Postulado de Euclides. Bien sabía él cuál concepto filosófico debe tenerse de esta verdad intuitiva, que al tratar de demostrarse presupone siempre definiciones implicitas que también necesitan demostración.

Porque Garavito, más que matemático, fue filósofo sincero que nunca tuvo en mira cosa diferente de la persecución de la verdad. Bien puede catalogársele entre los filósofos de que nos habla el doctor Duarte, cuando afirma en su escrito: "Los filósofos que no son matemáticos, como Lotze, han sido en general, enemigos acérrimos de la Metageometría. Lotze expresaba la esperanza de que la Filosofía no se dejara dominar en esta materia por las Matemáticas".

Garavito, al igual de Lotze, fue adverso a las Geometrías no-euclidianas, por concepto propio y no por principios de autoridad, ya que su conocimiento de los numerosísimos autores que han propugnado por el triunfo de las nuevas ideas geométricas, era muy limitado.

Nuestra ignorancia a este respecto, mucho mayor que la suya, nos ha hecho comprenderle por este aspecto y por eso podemos afirmar que el Profesor colombiano jamás hubo de preocuparse de la demostración del Postulado de Euclides.

Así procuraremos demostrarlo en un proximo número de esta Revista, en donde haremos ver que los errores que el doctor Duarte cree encontrar en las exposiciones de Garavito, no lo son para todos, por cuanto la escuela de los pangeómetras no es universal, ni las ideas contrarias a la matemática clásica han obtenido hasta ahora un triunfo absoluto. El antagonismo existente entre clásicos e innovadores en estas materias es, en nuestro pobre concepto, cuestión de temperamentos. El hablar del error en que están quienes no piensan como nosotros, sobre cuestiones que aún se discuten, es pronunciar fallos excátedra.

Por pensar así es que hemos procurado insertar en estas páginas los conceptos tan bien documentados del doctor Duarte, con el propósito de discutirlos de acuerdo con las doctrinas de nuestro venerado maestro, a quien seguimos con sincera convicción. Creemos con ello prestar un servicio a quienes se interesen por el serio estudio y gusten de la discusión serena y constructiva.

Que nuestro colega, quien, repetimos, nos merece profundo respeto e irrestricta admiracion, nos perdone esta explicación y acepte por anticipado la réplica que habremos de hacer próximamente con más estudio y mayor conocimiento del asunto, como prueba de que el trabajo a que nos referimos es digno, como ninguno, de la consideración de los estudiosos. 